Mens

revue d'histoire intellectuelle de l'Amérique française

\title{
L'iconographie rébellienne, la face cachée de l'historiographie des rébellions de 1837-1838 : quelques études de cas
}

\section{France St-Jean}

Volume 10, numéro 1, automne 2009

URI : https://id.erudit.org/iderudit/1023160ar

DOI : https://doi.org/10.7202/1023160ar

Aller au sommaire du numéro

Éditeur(s)

Centre de recherche en civilisation canadienne-française

ISSN

1492-8647 (imprimé)

1927-9299 (numérique)

Découvrir la revue

Citer cet article

St-Jean, F. (2009). L’iconographie rébellienne, la face cachée de

l'historiographie des rébellions de 1837-1838 : quelques études de cas. Mens, 10(1), 95-138. https://doi.org/10.7202/1023160ar
Résumé de l'article

Au contraire du sujet historique, l'iconographie des rébellions a suscité jusqu'ici bien peu d'intérêt de la part des chercheurs, et ce, bien qu'il s'agisse d'un vecteur significatif dans la construction de l'imaginaire collectif à l'égard de ce moment que l'on dit fondateur de l'histoire du Québec et du Canada. Ce regard attentif sur quelques-unes des œuvres qui composent ce corpus, produites tant au moment des insurrections qu'ultérieurement, en est d'abord un centré sur l'objet matériel. Ces œuvres remises par ailleurs dans leurs contextes de production et de première réception, leur analyse permet une autre lecture de la place occupée par le moment patriote dans la mémoire des générations qui les ont vues naître. 


\section{L'iconographie rébellienne, la face cachée de l'historiographie des rébellions de 1837-1838: quelques études de cas}

France St-Jean

Centre de recherche en civilisation

canadienne-française Université d'Ottawa

\section{Résumé}

Au contraire du sujet historique, l'iconographie des rébellions a suscité jusqu'ici bien peu d'intérêt de la part des chercheurs, et ce, bien qu'il s'agisse d'un vecteur significatif dans là construction de l'imaginaire collectif à l'égard de ce moment que l'on dit fondateur de l'histoire du Québec et du Canada. Ce regard attentif sur quelques-unes des œuvres qui composent ce corpus, produites tant au moment des insurrections qu'ultérieurement, en est d'abord un centré sur l'objet matériel. Ces œuvres remises par ailleurs dans leurs contextes de production et de première réception, leur analyse permet une autre lecture de la place occupée par le moment patriote dans la mémoire des générations qui les ont vues naître.

\section{Abstract}

Unlike the historical subject, the iconography of the 1837-1838 Rebellions has generated very little scholarly interest; and yet it constitutes a significant vector in the construction of the collective imaginary of what is said to be a founding moment in the history of Quebec and Canada. 
The present examination of various works produced both during and after the Rebellions is drawn from a larger corpus and is centred on material objects. Placed in the context of their production and first reception, an analysis of these works allows for a new reading of the place occupied by the Patriots in the memory of the generations that witnessed their creation.

Les rébellions bas-canadiennes de 1837-1838 occupent dans l'histoire du Québec, tout comme dans celle du Canada, une place toute particulière. Perçues comme moment significatif ayant mené à l'union du Haut et du Bas-Canada en 1841, puis, à la Confédération canadienne en 1867 , elles constituent, pour les uns, un moment phare de l'histoire du Québec, voire annonciateur du projet d'indépendance, alors que pour d'autres, elles sont l'élément déterminant qui a marqué la naissance de la société démocratique canadienne. En fait, depuis plus de cent soixante-dix ans, les insurrections ont suscité l'intérêt autant chez les historiens et les sociologues que chez les médiateurs de la mémoire patriote ${ }^{1}$. Les artistes ne sont d'ailleurs pas en reste. Quelques centaines d'œuvres et d'illustrations ${ }^{2}$ forment l'imposant corpus iconographique rébellien ${ }^{3}$. Des représentations qui sont tout autant le témoignage des acteurs de l'époque qu'une

\footnotetext{
${ }^{1}$ Pour avoir une idée de l'étendue des études abordant le sujet des rébellions, on consultera entre autres Gilles Laporte, "Introduction », dans Gérard Filteau, Histoire des patriotes, Sillery, Septentrion, 2003 [1938], p. XxxII-XxxIII; Jean-Paul Bernard, "Présentation", Bulletin d'histoire politique: les Rébellions de 1837-1838 au BasCanada, vol. 7, $\mathrm{n}^{\circ} 1$ (automne 1998), p. 11 et Jean-Paul Bernard, Les rébellions de 1837-1838, Montréal, Boréal Express, 1983, p. 347-349.

${ }^{2}$ Dans la discipline de l'histoire de l'art les termes œuvre et illustration ne sont pas équivalents. Le premier réfere généralement à l'unicité et à l'authenticité de l'objet (et ce, même s'il est reproduit), le second à sa reproductibilité inhérente. L'utilisation ici des deux termes rend compte du caractère complexe du corpus des représentations rébelliennes.

${ }^{3}$ Le corpus visuel rébellien s'étend bien au-delà puisqu'il comprend également l'ensemble de la filmographie abordant le sujet des rébellions ainsi que les œuvres tridimensionnelles telles les sculptures et les monuments commémoratifs.
} 
interprétation rétrospective sur le sujet. Pourtant, force est de constater qu'hormis la recension qu'a effectuée Robert-Lionel Séguin pour L'esprit révolutionnaire dans l'art québécois ou les articles de Daniel Vaillancourt et de Marianne Thibault à propos de l'aquarelle Un Vieux de '37 d'Henri Julien ${ }^{4}$, l'iconographie illustrant les insurrections demeure un objet d'étude négligé, que ce soit de la part des historiens des rébellions ou des historiens de l'art. Ce constat n'est cependant pas unique aux représentations des rébellions. Jean Pirotte souligne que même si l'historien n'a «jamais totalement ignoré les documents figurés; ceux-ci [...] n'apparaissent souvent que comme un appui illustratif des récits ${ }^{5}$. Dans son ouvrage Eyewitnessing: The Uses of Images as Historical Evidence, Peter Burke pousse plus loin la réflexion et soutient que

When they do use images, historians tend to treat them as mere illustrations, reproducing them in their books without comment. In cases in which the images are discussed in the text, this evidence is often used to illustrate conclusions that the author has already reached by other means, rather than to give new answers or to ask new questions ${ }^{6}$.

Présentes autant dans l'ouvrage savant que dans le discours commémoratif, les représentations visuelles illustrant les rébellions de 1837-1838 ont suscité, depuis leur création, de multiples et subséquents investissements idéologiques. Certaines d'entre elles sont

\footnotetext{
${ }^{4}$ Robert-Lionel Séguin, L'esprit révolutionnaire dans l'art québécois: de la déportation des Acadiens au premier conflit mondial, Montréal, Éditions Parti Pris, 1972, 579 p.; Daniel Vaillancourt, "Les têtes à Patriote: une figure retorse au xix" siècle", Voix et Images, vol. XXVI, ${ }^{\circ} 3$ (printemps 2001), p. 456-473 et Marianne Thibault, "Les représentations des Patriotes dans l'art québécois: le cas d'Henri Julien", Bulletin d'histoire politique: les patriotes de 1837-1838, vol. 12, $\mathrm{n}^{\circ} 1$ (automne 2003), p. 28-42.

${ }^{5}$ Jean Pirotte, «Écoute Clio!... Les images parlent. Ouvertures historiennes à l'analyse des documents iconiques", dans Jean-Louis Tilleuil, dir., Théories et lectures de la relation image-texte, Cortil-Wodon (Namur), Éditions modulaires européennes, 2005, p. 27. (Texte-Image)

${ }^{6}$ Peter Burke, Eyewitnessing: The Uses of Images as Historical Evidence, Ithaca, Cornell University Press, 2008 [2001], p.10.
} 
même devenues des incontournables de l'histoire nationale, cristallisant dans l'imaginaire collectif autant l'événement lui-même, issu du passé, que sa récupération, au présent, notamment dans le projet politique d'un Québec souverain?. Force est toutefois de constater que les lectures perceptuelles qui découlent de ces réappropriations dissimulent le vouloir dire original d'œuvres ou d'illustrations réalisées à une autre époque.

L'histoire de l'art au Québec étant somme toute une discipline encore jeune, il est plausible de penser que les interprétations ou les mésinterprétations ${ }^{8}$ véhiculées à propos de ces représentations relèvent d'une méconnaissance du corpus. Sans prétendre livrer ici une étude exhaustive de l'ensemble de l'imagerie rébellienne, j'entends me pencher sur le contexte de production', incluant l'iconographie, l'intention de l'artiste, celle des commanditaires dans certains cas et la première réception d'œuvres choisies. La perspective proposée ne se veut aucunement une relecture du fait historique. Néanmoins, il s'agit d'un regard différent, croisé pour utiliser un terme à la mode, sur la place occupée par les insurrections dans la mémoire collective; un regard centré sur l'objet matériel et la perception de celui-ci au moment où il est réalisé.

\footnotetext{
${ }^{7}$ Dans ma thèse de doctorat, j'ai montré comment les images du Patriote ont été (et sont encore) un vecteur important dans la médiation de la mémoire des rébellions. J'y ai aussi fait état des investissements, réinvestissements, voire travestissements dont les œuvres et les illustrations qui composent l'imagerie rébellienne ont été l'objet, et ce, dès le lendemain des insurrections. Pour certaines, ces multiples lectures ont contribué à leur mythification. France St-Jean, Images du Patriote : objets commémoratifs, intentions variables, thèse de doctorat (histoire de l'art), Montréal, Université du Québec à Montréal, 2009.

${ }^{8}$ Cela va de la date de production, au nom de l'artiste qui a créé l'œuvre ou l'illustration, en passant par le sujet représenté.

${ }^{9}$ L'évocation des contextes politiques et sociaux qui ont vu naître les œuvres et les illustrations représentant les rébellions semblera, pour le lecteur averti, manquer de nuances. Cela s'explique en raison de ma démarche, essentiellement centrée sur l'objet matériel, du nombre d'objets étudiés et de leur étalement dans le temps, d'autant que malgré le nombre restreint d'œuvres représentant les rébellions bascanadiennes présentées ici, mon corpus de recherche en compte au-delà de trois cents, couvrant cent soixante-dix ans d'histoire.
} 
Compte tenu du nombre élevé d'œuvres et d'illustrations composant le corpus iconographique rébellien, un choix s'imposait. D'une part, la sélection reflète l'état d'avancement de mes recherches ${ }^{10}$, d'autre part, les œuvres choisies m'apparaissent comme exemplaires de leur acception en tant qu'images de Patriote. Il importe de noter ici que je fais une distinction entre les termes æuvre et illustration et la notion d'image. Les premiers désignent l'objet matériel représentant ou interprétant un sujet, une opinion ou un état d'esprit. Le dernier, dont j'emprunte la définition aux études urbaines, plus particulièrement à l'idée (l'image) que l'on se fait de la ville, indique "une manifestation sensible d'un total syncrétique mais invisible, contingenté par l'imaginaire ${ }^{11}$ "; non plus celle de l'imaginaire de l'artiste qui crée une œuvre ou une illustration, mais bien la manifestation imaginative de la collectivité qui investit cette même œuvre ou illustration de référents symboliques qui lui sont propres.

Parmi les œuvres témoignages laissées par des acteurs des rébellions, j'ai choisi The Insurgents, At Beauharnois, Lower Canada (Quebec), une aquarelle réalisée par Katherine Jane Ellice (v. 18141864) en 1838, les trois aquarelles peintes par Robert-Shore-Milnes Bouchette (1805-1879) en 1837-1838 et représentant sa cellule dans la prison commune de Montréal, l'ensemble des portraits de patriotes dessinés par Jean-Joseph Girouard (1795-1855), aussi en 1837-1838, ainsi que les Lithographic Views tirées des esquisses de Lord Charles Beauclerk (1812-1861) et qui ont été publiées en 1840. Quant aux œuvres posant un regard rétrospectif sur les insurrections, elles ne sont qu'au nombre de trois: Manifestation des Canadiens contre le

\footnotetext{
${ }^{10}$ Bien que dans ma thèse j'aie présenté l'ensemble du corpus rébellien et les multiples lectures perceptuelles que chacune des ouvres et des illustrations a suscitées, il n'en demeure pas moins que pour l'essentiel, l'analyse contextuelle de création des œuvres, les recherches restent à faire.

${ }^{11}$ Cette définition de l'image est empruntée à Lucie K. Morisset, Luc Noppen et Denis Saint-Jacques, dir., "Entre la ville imaginaire et la ville identitaire: de la représentation de l'espace ", dans Ville imaginaire, Ville, identitaire: échos de Québec, Québec, Nota Bene, 1999, p. 5.
} 
gouvernement anglais, à Saint-Charles, en 1837, une huile sur toile peinte par Charles Alexander (1864-1915) en 1891, la maquette pour le monument Chénier réalisée par Louis-Philippe Hébert (18501917) en 1894, et Papineau addressing meeting, une aquarelle de Charles William Jefferys (1869-1951) produite vers 1912. En tout, sept œuvres ou ensemble d'œuvres seront étudiées, mettant ainsi au jour le vouloir dire originellement inscrit dans la matière picturale.

Vecteurs d'un imaginaire fluctuant selon les époques et les perspectives adoptées, ces œuvres ont été maintes fois reproduites, voire déconstruites, afin de soutenir un tout autre discours que celui qui leur était associé au moment de leur création. C'est le cas notamment de The Insurgents, At Beauharnois, Lower Canada (Quebec) et de Manifestation des Canadiens contre le gouvernement anglais, $\grave{a}$ Saint-Charles, en 1837. Ces deux œuvres ont été incluses dans le dispositif muséal de la Prison-des-Patriotes, lieu de mémoire des rébellions de 1837 et $1838^{12}$. De la première, on ne présente qu'un détail, réduisant la portée significative de l'œuvre, alors qu'on a privilégié l'effet miroir pour la seconde, ce qui a pour résultat de décupler l'assistance de cette fameuse assemblée. Utilisée pour la promotion de la Journée nationale des patriotes, Papineau addressing meeting, quant à elle, ne se reconnaît plus que par certains fragments, éliminant par la même occasion certains éléments iconographiques, notamment l'église.

La prégnance dans l'imaginaire visuel des rébellions de la maquette pour le monument à Jean-Olivier Chénier relève d'un tout autre processus. Malgré la décision du comité du monument Chénier de confier la réalisation de la statue à un sculpteur américain, le projet proposé par Louis-Philippe Hébert demeura ancré dans la mémoire collective grâce à la diffusion d'un dessin largement inspiré du

${ }^{12}$ Ce lieu de mémoire dédié à l'histoire des patriotes a ouvert ses portes en 2003. Il est situé dans les locaux du siège social de la Société des alcools du Québec où, anciennement, se trouvait la prison commune du district de Montréal. 
monument. Réalisé par Albert Fournier ${ }^{13}$, ce dessin représentant le leader patriote illustra la page de garde des romans Le Patriote et L'Espion des Habits rouges de Jean Féron (pseudonyme de JosephMarc-Octave Lebel), publiés respectivement en 1926 et 1928 aux Éditions Édouard Garand. En 1971, le dessin de Fournier fut de nouveau reproduit, cette fois dans l'opuscule Culture et libération nationale. Il était alors mis en parallèle avec la «Déclaration politique soumise aux membres et sympathisants du CDDP [Comité pour les droits démocratiques du peuple], aux organisations patriotiques, aux masses du Québec, par la Branche de Propagande Chénier du CDDP (21 janv. 71) pour approbation dans la lutte ${ }^{14}$ ". Depuis quelques années, la maquette d'Hébert, la source d'inspiration du dessin, a repris place dans l'imaginaire collectif. Elle a d'ailleurs été incluse dans deux expositions abordant la question des rébellions, la première, temporaire, 1837-1838 Rébellions - Patriotes VS Loyaux, qui s'est tenue à Pointe-à-Callière, musée d'archéologie et d'histoire de Montréal en 2007 et 2008, la seconde, La rébellion de 1837 à SaintEustache, que l'on peut consulter sur le site du Musée virtuel du Canada, sous l'onglet "Histoire de chez nous".

Les dessins de Girouard et les Lithographic Views de Beauclerck méritent que l'on s'y attarde, et ce, même si elles n'ont pas fait l'objet de réappropriation. CEuvres incontournables, elles sont parmi les représentations des patriotes qui ont servi à illustrer et illustrent encore régulièrement le récit historique et la mise en mémoire des rébellions. Elles ont permis d'accoler un visage à des noms de patriote et de voir le déroulement des batailles de Saint-Charles et de SaintEustache, et ce, même si on souligne régulièrement que les points de

\footnotetext{
${ }^{13}$ On connaît peu de chose au sujet d'Albert Fournier, pas même les dates de naissance et de décès, sinon qu'il a été le principal collaborateur d'Édouard Garand. Sa production visuelle semble toutefois avoir été exclusivement destinée aux ouvrages édités par ce dernier.

${ }^{14}$ Culture et libération nationale, vol 1, no 1 (été 1971), p. 9. Service des archives et de gestion des documents, UQAM, Collection de publications de groupes de gauche et de groupes populaires (21P-900:03/39).
} 
vue illustrés par Beauclerk s'écartent de la réalité. Enfin, il était tout aussi important de discuter des aquarelles de Bouchette, ne serait-ce qu'en raison de l'attention que l'on a portée ces dernières années à la prison où furent emprisonnés les patriotes et où douze d'entre eux furent pendus. Leur étude offre non seulement un regard sur la disparité de traitement des prisonniers de l'époque, mais rend compte également d'une réalité qui, sans s'y opposer, diffère quelque peu de celle proposée dans les récentes œuvres cinématographiques abordant les rébellions.

L'aquarelle d'Henri Julien, Un Vieux de '37, est sans doute l'œuvre la plus connue de l'imagerie rébellienne, notamment en raison de sa récupération politique, sous une forme simplifiée, par le Front de libération du Québec (FLQ) lors de la crise d'Octobre $1970^{15}$. Considérée comme le symbole des rébellions, voire l'allégorie synthétique de l'ensemble des événements qui ont marqué le Québec, elle assure depuis plus d'une trentaine d'années une présence indéfectible dans le paysage commémoratif des rébellions et joue le rôle de porteétendard des revendications indépendantistes. Comme je le soulignais précédemment, c'est aussi une des seules œuvres ou illustrations ayant suscité autant d'intérêt pour le vouloir dire inscrit dans le pictural. Mon objectif n'étant pas d'offrir une étude exhaustive, ni même une vue globale de l'ensemble des représentations des rébellions en tant qu'objet matériel, et m'étant déjà beaucoup attardée à Un Vieux de '37.16, j'ai privilégié celles qui ont suscité peut-être moins d'attention mais qui, néanmoins, sont tout aussi importantes.

${ }^{15}$ À la suite de l'enlèvement, le 5 octobre 1970, du diplomate britannique James Richard Cross, la cellule «Libération » du Front de libération du Québec (FLQ) faisait parvenir aux médias un communiqué sur lequel la silhouette d'Un Vieux de '37 était reproduite en filigrane.

${ }^{16} \mathrm{St}$-Jean, Images de Patriote: objets commémoratifs, intentions variables, particulièrement les chapitres III et V. Lire également, France St-Jean, "La lecture de la mémoire à travers le repère visuel : le cas du patrimoine commémoratif rébellien ", dans Anne-Marie Broudehoux, dir., Paysages construits: mémoire, identité, idéologies, Montréal, Éditions MultiMondes, 2006 p. 73-90. De la même auteure, voir aussi "Un Vieux de '37: transcription visuelle d'un regard sur les rébellions ou 


\section{Témoignages visuels des acteurs des rébellions}

Les premières œuvres réalisées représentant les rébellions remontent à l'époque des événements eux-mêmes. Jusqu'à maintenant, 118 œuvres et illustrations ${ }^{17}$ réalisées entre 1830 et 1840 ont été répertoriées, soit le tiers de l'ensemble du corpus inspiré de cette page d'histoire. Elles sont autant le résultat du regard britannique que du regard canadien (dénominatif par lequel on désignait les habitants du BasCanada à l'époque).

La majorité de ces œuvres dépeignant les acteurs, surtout les patriotes, la tension politique avant la prise d'armes, les scènes de bataille ou encore les lieux d'emprisonnement, qui sont aujourd'hui largement connues, n'étaient pas à l'origine destinées à un usage public. C'est le cas notamment des œuvres de Katherine Jane Ellice et de Robert Shore-Milnes Bouchette. Emprisonnés tous deux, la première au presbytère de Beauharnois par les patriotes en novembre 1838 , le second à la prison commune de Montréal sous l'ordre du colonel Colborne à la fin de l'automne 1837 et au début de l'hiver 1838 , ils mirent à profit leur talent de peintre amateur afin de rendre compte visuellement des lieux de leur détention. C'était là une façon d'instruire leurs proches de leur situation personnelle. The Insurgents, At Beauharnois, Lower Canada (Quebec) 1838, bien connue des amateurs de bière puisqu'elle figure sur l'étiquette de 1837 de la brasserie Unibroue ${ }^{18}$, ne semble avoir été découverte ${ }^{19}$ que dans les

actualisation de l'identité canadienne française héritée du colon français? ", dans Monika Boehringer, dir., (Se)Voir et savoir: constructions identitaires dans les espaces littéraire et pictural de l'Acadie et du Québec, Moncton, Institut d'études acadiennes (à paraître) ainsi que "Représentations visuelles et territoires identitaires: le cas de deux "images" du Patriote dans le récit historique Canada/Québec ", dans Jeanette den Toonder, dir., Redécouvrir l'espace canadien, Groningen (Pays-Bas), Barkhuis Pers (à paraître) (Série ACSN).

${ }^{17}$ Il s'agit ici des œuvres incluses dans le répertoire accompagnant ma thèse de doctorat.

${ }^{18}$ Cette bière est brassée depuis 1997.

${ }^{19}$ On lira avec intérêt l'article d'Yves Bergeron, "L'astrolabe de Champlain : parcours d'un objet mythique du patrimoine canadien ", Encyclopédie du patrimoine de 
années 1960, au moment où les aquarelles illustrant le Diary d'Ellice furent retirées du manuscrit et transférées à la division de l'art documentaire des Archives nationales du Canada (maintenant Bibliothèque et Archives Canada). Les aquarelles de Robert-Shore-Milnes Bouchette ne sont quant à elles venues enrichir les collections muséales et d'archives publiques qu'à partir de 1956. La première exposition incluant l'une d'elles, Bouchette en prison 1838, fut présentée au Musée de la Province de Québec (aujourd'hui le Musée national des beaux-arts du Québec) trois ans après. Il en est également de même pour les portraits "souvenirs ${ }^{20}$ " dessinés par Jean-Joseph Girouard. Destinés originalement aux familles des patriotes détenus à la prison commune de Montréal ${ }^{21}$, ils prirent rapidement place dans le domaine public. Dès 1873, plusieurs d'entre eux illustrèrent les panégyriques que signa Laurent-Olivier David pour L'OpinionPublique $e^{22}$. D'autres

l'Amérique française, 2009, http://www.ameriquefrancaise.org, consulté le 27 juin 2009. Il y décrit comment le moment même de la découverte d'une œuvre, même s'il est contestable, contribue à sa notoriété et, éventuellement, à sa patrimonialisation.

${ }^{20}$ C'est ainsi que Gérard Morisset les qualifiait dans ses lettres datées du 10 août 1959 et du 17 septembre 1957 adressées à Jeanne Girouard-Décarie. Cité dans Clément Laurin, Jean-Joseph Girouard et les Patriotes de 1837-38: portraits, Montréal, Bibliophile du Canadiana et Osiris, 1973, p. 3.

${ }^{21}$ Les œuvres ont été peintes durant l'incarcération de Girouard, d'abord entre le 26 décembre 1837 et le 16 juillet 1838 puis, entre le 4 novembre et le 27 décembre 1838. Alain Messier, Dictionnaire encyclopédique et historique des patriotes $1837-$ 1838, Montréal, Guérin, 2002, p. 213'et 249.

${ }^{22}$ Le dépouillement de L'Opinion Publique a été fait du 15 février 1877 jusqu’à la fin décembre 1880. Il s'écoulait parfois plusieurs semaines, voire plusieurs mois entre la publication des articles. Quant à l'en-tête, il se déclinait autant sous "Événements de 37-38", "Les Hommes de 37-38" ou encore "Les Hommes de 37 ", ne laissant parfois place qu'au seul titre tel: "Les prisonniers de 37 ». Après le 25 mars 1880, nous n'avons relevé aucune gravure qui aurait été tirée des dessins de Jean-Joseph Girouard. L'ensemble de ces articles fit l'objet d'une publication ultérieurement, en 1884, sous le titre: Les Patriotes de 1837-1838. Il s'agit là d'un des ouvrages que consacra David à la réhabilitation des patriotes. Mentionnons aussi Jean-Olivier Chénier, le héros de Saint-Eustache (vers 1893) et Les deux Papineau (1896). 
furent reproduits dans le quotidien $L a$ Presse $e^{23}$ en 1924, à l'intérieur de la série «La tragique épopée des patriotes de 1837-38" tirée du Journal d'un Fils de la Liberté, réfugié aux États-Unis, par suite de l'nsurrection Canadienne, en 1837 de Louis-Joseph-Amédée Papineau. Cette diffusion relativement soutenue ${ }^{24}$ des œuvres de Girouard contribua à fixer dans l'imaginaire collectif les visages patriotes.

Seules les Lithographic Views de Charles Beauclerk s'adressaient, dès leur réalisation, à un large public. Publiées à. Londres et destinées initialement à l'aristocratie, celles-ci trouvèrent néanmoins une place de choix dans les collections d'amateurs de canadianas, autant au Canada qu'aux États-Unis. Une réédition de l'ouvrage de Beauclerk

${ }^{23}$ Cette série parut entre le 5 janvier et le 24 mai 1924, parallèlement à la campagne de souscription qui avait cours afin que soit érigé sur le site de l'ancienne prison commune de Montréal un monument à la mémoire des patriotes qui y furent pendus.

${ }^{24}$ C'est à Clément Laurin que l'on doit de connaitre l'ensemble de la production de cet artiste patriote. En 1973, il a réuni dans un seul ouvrage, Jean-Joseph Girouard et les Patriotes de 1837-38: portraits, la plupart des dessins de Girouard qui, jusque-là, avaient été conservés par ses descendants. Conjointement à la publication de cet ouvrage, les œuvres furent exposées du 25 au 27 novembre 1973 à la Salle Dorée du Sheraton-Mont-Royal dans le cadre du troisième Salon du livre ancien de Montréal. Il faut ajouter à ce corpus les «images " numérisées tirées de L'Opinion publique et mises en ligne par Bibliothèque et Archives nationales du Québec (2002) (ces images sont tirées de L'Opinion publique (1870-1883), de la revue Le Monde illustré (1884-1902, 1907) et de L'Album universel (1902-1907). On les trouve sous l'onglet "Revues d'un autre siècle " dans la section "Autres images" de la Collection numérique de Bibliothèque et Archives nationales du Québec, http://www4.bnquebec.ca/illustrations/accueil.htm, consulté le 21 août 2006). Ces images, suivies de "L'intégral des dessins de patriotes exécutés par Jean-Joseph Girouard " sur le site Les Patriotes de 1837@1838 (2006), concoururent à sortir de l'anonymat une part importante de l'imagerie rébellienne, tout en permettant de donner un visage aux patriotes. (L'ensemble des images représentant les patriotes a été importé sur le site Les Patriotes de 1837@1838: Les Rébellions du Bas-Canada, http://cgi2.cvm.qc.ca/glaporte/1837.pl?out=article\&pno=1045\&cherche=IMAG ES, consulté le 21 août 2006.) Bien que nous ayons relevé quelques erreurs quant à la correspondance nom/visage, cet « intégral » demeure un outil important pour l'identification des hommes qui participèrent aux rébellions de 1837-1838. 
fut d'ailleurs publiée par Mika Publishing en 1980 afin de pallier la rareté des exemplaires disponibles sur le marchée 25 .

\section{The Insurgents, At Beauharnois, Lower Canada (Quebec), 1838}

C'est durant son séjour dans les Canadas en 1838, alors qu'elle accompagnait son mari Edward Ellice fils, secrétaire particulier de Lord Durham, que la Britannique Katherine Jane Ellice a peint The Insurgents, At Beauharnois, Lower Canada (Quebec) ${ }^{26}$. Suivant la mode de l'époque, et acquiesçant par la même occasion à la demande de son beau-père, elle tint un journal de voyage qu'elle agrémenta d'aquarelles illustrant ses déplacements et maints détails de sa vie dans la colonie. Celles-ci ne sont à ses yeux «Nothing however but what I call scrabbles-recollections of Beauharnois which I shall like to look at when we go home ${ }^{27 ! " ~}$

The Insurgent, At Beauharnois, Lower Canada (Quebec) est toutefois bien différente de l'ensemble des «scrabbles" que ramena Ellice en Angleterre. Plus qu'un souvenir visuel de sa captivité, cette aquarelle représente, au-delà des "picturesque ruffians ${ }^{28}$ " qui montaient la garde autour du presbytère le 7 novembre $1838^{29}$, l'état d'impuissance et

${ }^{25}$ Lors de la vente de la collection Rosanna Seaborn au Ritz-Carleton en 2004, un exemplaire de Lithographic Views of Military Operations in Canada se serait vendu pour la somme de 3800 \$. Rosanna Seaborn collectionnait les ouvrages sur les rébellions de 1837-1838 depuis 50 ans. Les recettes de cette vente devaient servir à financer son projet de film sur cette page de l'histoire du Québec. Les Patriotes de 1837@1838, http://cgi2.cvm.qc.ca/glaporte/1837.pl?out=article\&pno=110 18 cherche=Bleury, consulté le 22 octobre 2006.

${ }^{26}$ Aquarelle sur mine de plomb sur papier vélin bleu-gris, $23,8 \mathrm{~cm} \times 16,6 \mathrm{~cm}$, Bibliothèque et Archives Canada (1990-215-24R).

${ }^{27}$ Jane Ellice, The Ellice Diary. Sunday, November 4, 1838 - Monday, November 12, 1838, Bibliothèque et Archives Canada, Fonds Ellice Family, MG24, série A2, vol. 50, October 13, 1838. Cité dans Michael Bell et Archives publiques du Canada, Painters in a New Land: from Annapolis Royal to the Klondike, Toronto, McClelland and Stewart; 1973, p. 13.

${ }^{28}$ Patricia Godsell, The Diary of Jane Ellice, Ottawa, Oberon Press, 1975, p. 140. Voir aussi Jane Ellice, The Ellice Diary, p. 170-305.

${ }^{29}$ Ellice spécifie clairement dans son journal, à l'entrée du 7 novembre 1838, qu'elle peint à partir d'une fenêtre de la chambre du presbytère où elle est retenue captive, les patriotes se trouvant à l'extérieur, afin de sauvegarder leur mémoire. 
de peur dans lequel elle se trouvait. Ses allusions au régime de terreur que mena Robespierre au lendemain de la prise de la Bastille en 1789 auprès des nobles français, parmi lesquels plusieurs furent exécutés sans procès, sont nombreuses dans son journal. L'analogie entre les insurrections bas-canadiennes et la Révolution française se traduit également dans la composition formelle de l'œuvre. La répétition des motifs (les armes employées par les patriotes) et l'alignement des personnages dans un cadrage serré rappellent étrangement les gravures éditées durant cette épisode de l'histoire de France. Selon Fabrice Bensimon ${ }^{30}$, dans l'Angleterre des années 1830, et peut-être plus particulièrement chez l'aristocratie, on commençait à peine à réhabiliter 1789. Longtemps, écrit-il, citant le journaliste britannique Walter Bagehot (1826-1877), «[1]a Révolution française effraya la nation anglaise; elle hanta tant les gens de cette génération qu'ils ne pouvaient regarder où que ce soit, sans en voir les traces ${ }^{31}$ ". Cet "écho de la Révolution française $e^{32}$ " se confirme visiblement dans The Insurgent, At Beauharnois, Lower Canada (Quebec), et ce, bien qu'Ellice soit née plus de vingt ans après la Révolution.

\section{Portraits de patriotes, $1837-1838$}

Notaire, homme politique et ardent patriote issu d'une famille d'artistes, Jean-Joseph Girouard a été initié très tôt par son cousin Thomas Baillargé aux rudiments du dessin, de la sculpture et de l'architecture. Les dessins qu'il a réalisés au moment des insurrections, particulièrement les portraits, doivent être considérés autant comme un témoignage patriote que l'énoncé des changements sociaux propres à cette époque.

\footnotetext{
${ }^{30}$ Fabrice Bensimon, «L'écho de la Révolution française dans la Grande-Bretagne du XIX siècle (1815-1870) ", Annales historiques de la Révolution française, $\mathrm{n}^{\circ} 342$. Article en ligne à l'adresse http://ahrf.revues.org/document 1942. html?format=print, consulté le 10 juin 2009.

${ }^{31} \mathrm{Ibid}$.

${ }^{32}$ Ibid.
} 
Au moment où éclatèrent les rébellions, le portrait, en tant que genre artistique, était en plein essor au Bas-Canada. Cette popularité concorde d'ailleurs avec les changements politiques et économiques que connaît la colonie en ce début de xIX $^{e}$ siècle ainsi qu'avec l'émergence d'une classe moyenne d'expression française. Qu'il soit destiné à la pièce d'apparat (portrait officiel ou professionnel) ou pour un usage plus personnel (miniature), le portrait constituait aux yeux du sujet peint (ou de son commanditaire dans le cas des portraits familiaux), et pour ceux qui le regardaient, la preuve tangible de son statut et de la position qu'il occupait dans la hiérarchie sociale. De format modeste ${ }^{33}$, ceux qu'a dessinés Girouard reflètent, bien sûr, le caractère intimiste que sous-tend leur réalisation dans l'enceinte de la prison commune de Montréal. Ils permettent aussi, tout comme ceux réalisés dans un contexte pacifique, de rendre compte de l'identité sociale des patriotes (médecin, avocat, notaire, arpenteur, marchand, aubergiste, instituteur, libraire, cultivateur, charpentier, imprimeur, journaliste ou peintre). En immortalisant sur papier vélin le profil ${ }^{34}$ des patriotes, Girouard offrait non seulement un souvenir aux familles et proches des prisonniers, mais il rendait compte de la place qu’occupaient désormais les Canadiens dans la société bas-canadienne et affirmait l'importance du mouvement patriote dans le Bas-Canada.

\section{Aquarelles de Bouchette, 1837-1838}

Moins utilisées dans la médiation de la mémoire des patriotes, les aquarelles qu'a peintes l'avocat et cartographe Robert-Shore-Milnes Bouchette et qui représentent sa cellule à la prison commune du district de Montréal sont, quant à elles, peut-être moins un regard sur les rébellions qu'une illustration des disparités dans les conditions de détention que l'on constatait, encore, à cette époque. Dans une

\footnotetext{
${ }^{33}$ Le format varie de $17,7 \mathrm{~cm} \times 21,5 \mathrm{~cm}$ à $27,3 \mathrm{~cm} \times 23,0 \mathrm{~cm}$.

${ }^{34}$ Bien que la plupart des dessins des patriotes soient des profils, il y a quelques portraits de trois quarts dont ceux de Wolfred Nelson et Louis-Hippolyte Lafontaine.
} 
moindre mesure, elles sont aussi un état des lieux de cette prison, dont la construction, débutée en 1832, ne se termina qu'en 1840 .

Il importe de rappeler ici, qu'au moment de son édification ${ }^{35}$, la nouvelle prison de Montréal, aussi appelée Prison Neuve, était l'une des rares traductions architecturales canadiennes ${ }^{36}$ de ce que Luc Noppen appelle l' «éveil des consciences ${ }^{37}$ ». Georges Blaicklock (1792-1828), à qui on avait confié la conception de la nouvelle prison $^{38}$, mit à profit les réflexions de penseurs tels Cesare Bonesana Beccaria $^{39}$, Jean-Jacques-Philippe Vilain ${ }^{40}$ et, plus particulièrement, John Howard ${ }^{41}$. Il proposa un bâtiment dont la répartition et l'aménagement des cellules devaient tout autant améliorer les

${ }^{35}$ L'achat des terrains se fit en novembre 1830 et l'appel d'offres pour la construction publié dans La Minerve le 21 février 1831. Les contrats furent accordés au début de l'année 1832. Luc Noppen, Dossier d'inventaire architectural de la Prison du Pied-du-Courant à Montréal, Québec, Ministère des Affaires culturelles, 1976, p. 33 ainsi que p. 83 , note 54 et p. 84 , notes 64 à 67.

${ }^{36}$ Le pénitencier de Kingston (1835) est le second qui, au Canada, s'inspira de la réflexion et des recommandations éditées plus de cinquante ans auparavant. Contrairement à la prison commune de Montréal, qui reprend le système de Philadelphie, le pénitencier de Kingston est inspiré du modèle de la prison d'Auburn (1816), dans l'État de New York. Les cellules sont adossées l'une à l'autre sur deux rangées au centre de l'édifice. Les couloirs, de part et d'autre du bloc cellulaire, longent les murs extérieurs.

${ }^{37}$ Noppen, Dossier d'inventaire architectural, p. 117.

${ }^{38}$ Blaicklock étant décédé prématurément, c'est à l'architecte John Wells (1790-1864) que fut confiée la réalisation du projet.

${ }^{39}$ Cesare Bonesana Beccaria est un économiste et un criminaliste italien. Son Traité des délits et des peines est paru en 1764. Il est disponible en ligne sur le site Les classiques des sciences sociales de l'UQAC, http://dx.doi.org/doi:10.1522/cla.bec. tra, consulté le 10 septembre 2007.

${ }^{40}$ Le Mémoire sur les moyens de corriger les malfaiteurs et les fainéants à leur propre avantage et de les rendre utiles à l'État de Jean-Jacques-Philippe Vilain fut présenté aux États de Flandres en 1771 et 1775.

${ }^{41}$ L'ouvrage de John Howard, The State of Prisons in England and Wales, parut en 1784. On consultera également le site Internet de la John Howard Society of Canada, http://www.johnhoward.ca/bio.htm, consulté le 6 septembre 2007. 
conditions de détention que permettre la réhabilitation des prisonniers. Les résultats ne semblent toutefois pas avoir été au rendez-vous ${ }^{42}$.

Du moins, c'est ce que l'on déduit à la lecture de la correspondance des patriotes. Présence de vermine (punaises, puces, poux, souris, rats), alimentation en eau déficiente, insalubrité des lieux, literie insuffisante, mauvaise ventilation et chauffage inadéquat sont au nombre de leurs doléances. Jean-Joseph Girouard, à qui l'on doit un plan de la prison, écrivait même que

[c]e bâtiment est un de nos édifices publics les plus mal faits [...]. Rien de plus absurde et de plus malcommode que les distributions de cette prison. [...] Ces messieurs [les commissaires responsables de la construction de l'édifice], par exemple, avaient été assez stupides que de s'imaginer que l'on pouvait chauffer un édifice comme celui-ci avec de l'air chaud par tuyaux circulant et tournant dans les diverses galeries ${ }^{43}$.

Au moment où les patriotes furent emprisonnés, la construction de la prison était loin d'être terminée. Ce n'est d'ailleurs qu'en 1839 qu'on installa l'éclairage au gaz et que tous les prisonniers disposèrent d'un lit, et ce, bien que dès 1836, on y admettait des détenus. C'est dire l'état dans lequel était la prison lorsqu'on amena les nombreux patriotes durant les automnes 1837 et 1838 . À titre indicatif, pour la seule année de $1837^{44}, 892$ hommes et 180 femmes y étaient emprisonnés ${ }^{45}$. Un nombre qui dépassait largement la capacité d'accueil de la prison, deux cents prisonniers tout au plus, prévue aux plans par Blaicklock.

${ }^{42}$ Dans son Dossier d'inventaire architectural, Luc Noppen mentionne que dès les années 1840, d'abord sous le gouvernement d'Union des Canadas, puis sous celui de la province de Québec, la réforme pénitentiaire « mit en évidence les lacunes de l'édifice de 1832-36». Noppen, Dossier d'inventaire architectural, p. 39.

${ }^{43}$ Extrait d'une lettre de Jean-Joseph Girouard à sa femme, datée du 21 mai 1838. Tirée de Georges Aubin, Au Pied-du-Courant, Lettres des prisonniers politiques de 1837-1839, Montréal, Comeau \& Nadeau, 2000, p. 125-127.

${ }^{44}$ C'est près de 500 patriotes qui furent incarcérés après les insurrections de 1837 et 800 après celles de 1838 .

${ }^{45}$ Noppen, Dossier d'inventaire architectural, p. 191. 
La lecture iconographique des aquarelles ${ }^{46}$ de Robert-ShoreMilnes Bouchette donne toutefois une tout autre interprétation de ces conditions de détention. Tant dans Bouchette en prison, $1838^{47}$ et Les Captifs, $1838^{48}$, que dans Imprisonment de R.S.M. Bouchette,

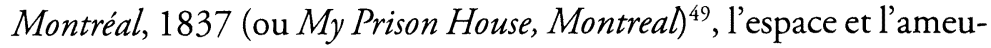
blement (un lit suspendu, une table de travail, une console, deux chaises et même un poêle personnel) mis à la disposition du prisonnier contrastent avec la petitesse et le simple grabat (et encore!) dont se contentait la majorité des détenus patriotes. Certes l'état de santé du patriote (Bouchette avait été blessé lors de l'escarmouche de Moore's Corner et il était fiévreux à son arrivée à la nouvelle prison), combiné au nombre élevé de prisonniers incarcérés, avaient certainement contribué à ce qu'il soit logé si avantageusement. Un extrait de ses Mémoires permet, mis en parallèle avec les aquarelles, de se faire une idée plus juste de cette cellule:

Je décorai ma chambre au moyen de mon pinceau et en reproduisant sur les murs des paysages qui se trouvaient dans mes cartons. D'un côté, je peignis les chutes Montmorency, de l'autre la villa de M. Atkinson, au Cap-Rouge. Autour de ma fenêtre cintrée s'enlaçaient une vigne et un chèvrefeuille. Les deux autres pans, car ma chambre affectait la forme d'un pentagone irrégulier, étaient occupés, l'un par des tablettes où j'avais placé mes livres, l'autre par ma petite batterie de cuisine, mon service de table, ma cafetière, etc., le tout autant que possible dissimulé et disposé de

${ }^{46}$ Il existe un autre dessin de Bouchette, The Wounded Captive Knight, conservé au Musée McCord d'histoire canadienne. Selon Denis Martin, ce dessin présenterait un point de vue différent de celui des aquarelles, d'un autre angle de la pièce. Il serait également beaucoup plus schématisé que les autres représentations que nous connaissons. Voir Denis Martin, "Robert-Shore-Milnes Bouchette 18051879 ", dans Mario Béland, dir., La peinture au Québec 1820-1850, nouveaux regards, nouvelles perspectives, Québec, Musée du Québec et Les Publications du Québec, 1991, p. 212.

${ }^{47}$ Aquarelle et encre sur papier, $13 \mathrm{~cm}$ x $18 \mathrm{~cm}$, Musée national des beaux-arts de Québec (56.10).

${ }^{48}$ Aquarelle sur papier, $15,2 \mathrm{~cm}$ x $16,6 \mathrm{~cm}$, Musée national des beaux-arts de Québec (56.302).

${ }^{49}$ Aquarelle et crayon sur papier vélin, $12 \mathrm{~cm}$ x 15,2 cm, Bibliothèque et Archives Canada (1970-083-2). 
manière à ne pas nuire à l'harmonie générale. Dans un coin de la pièce, je placai $[s i c]$ mes portefeuilles, dans l'autre ma guitare. Le plafond, naturellement, devait être bleu ciel ${ }^{50}$. Il n'est cependant pas le seul à avoir bénéficié de tels privilèges, Wolfred Nelson eut aussi droit à une pièce plus grande que le cachot dans lequel il avait été jeté à son arrivée. Est-ce en raison du dévouement de son père, Joseph, envers la couronne britannique ${ }^{51}$ que Robert-Shore-Milnes Bouchette eut droit à ces égards? Ou tout simplement parce qu'il avait les moyens financiers d'obtenir de tels privilèges $^{52}$ ? On ne saurait répondre aussi clairement à ces questions. Néanmoins, il est indéniable qu'il y avait, encore à cette époque, même à la toute nouvelle prison, des disparités dans les conditions de détention et que certains leaders patriotes surent en profiter.

\section{Lithographic Views, 1840}

Charles Beauclerck avait, à l'instar des officiers britanniques ${ }^{53}$ issus de la Royal Military Academy de Woolwich, des notions en topographie militaire et en dessin de paysage. Ce qui lui permit

${ }^{50}$ Cité dans Martin, "Robert-Shore-Milnes Bouchette 1805-1879». Voir aussi les Mémoires de Robert-S.-M. Bouchette 1805-1840, recueillis par son fils Errol Bouchette, annotés par Alfred DuClos DeCelles, Montréal, La Cie de publication de la Revue Canadienne, 1903, disponible à l'adresse url suivante: http://classiques. uqac.ca/classiques/bouchette_robert_sm/memoires_robert_sm_bouchette/ memoires_rsm_bouchette.pdf, consulté le 23 août 2006.

${ }^{51}$ Joseph Bouchette était l'arpenteur général du Bas-Canada. Il avait été nommé à ce poste en 1804 sur l'insistance du gouverneur Sir Robert Shore Milnes. Désireux sans doute de prouver sa loyauté, l'année suivante il choisit Milnes comme parrain de son plus jeune fils. Tout au long de sa carrière de fonctionnaire, Joseph Bouchette a soutenu les idées du parti des bureaucrates, participant notamment à la création d'un comité pour l'union des Canadas. Dictionnaire biographique du Canada en ligne, entrée "Joseph Bouchette", http://www.biographi.ca, consulté le 12 juin 2009.

${ }^{52} \mathrm{Il}$ avait même été autorisé à retenir les services d'un jeune garçon, lui-même emprisonné pour quelques méfaits sans aucun lien avec les insurrections. C'est d'ailleurs ce même garçon qui lui avait fait cadeau de l'oiseau représenté sur chacune de ses aquarelles. Mémoires de Robert-S.-M. Bouchette, p. 60.

${ }^{53}$ On pense notamment à George Heriot, Philip John Bainbrigge et James Duncan. 
d'acquiescer à la "particular request of friends ${ }^{54}$ » et de faire paraitre à Londres son Lithographic Views of Military Operations in Canada under His Excellency Sir John Colborne, During the Late Insurrection from Sketches by Lord Charles Beauclerk; Accompanied by Notes Historical and Descriptive. Son récit, qui relate aussi bien les rébellions qui se déroulèrent sur le territoire des Canadas que ses expéditions personnelles le menant des bords du Saint-Laurent jusqu'aux forêts au nord du fort militaire de Kingston, est à inclure dans la tradition des ouvrages militaires illustrés et des récits de voyage qui étaient fort appréciés de l'aristocratie britannique ${ }^{55}$.

Toutefois, bien qu'il ait décrit l'ensemble des opérations menées par l'armée britannique, de l'escarmouche qui eut lieu sur le chemin Chambly, sur la Rive-Sud de Montréal, jusqu'aux insurrections menées par les rebelles du Haut-Canada, en passant par la bataille de SaintDenis, il n'illustra que l'affrontement qui eut lieu à Saint-Charles le 25 novembre 1837, incluant la campagne militaire qui l'a précédée, et celui de Saint-Eustache, le 14 décembre de la même année. En tout, il a réalisé six lithographies (Passage of the Richelieu by Night, 22nd November, 1837; Colonel Wetherall's Bivouac at St. Hilaire de Rouville, 23rd, 24th November, 1837; A Fortified Passage - Colonel

\footnotetext{
${ }^{54}$ Lord Charles Beauclerk, "Preface», Lithographic Views of Military Operations in Canada Under His Excellency Sir John Colborne, G.C.B., during the late Insurrection from sketches by Charles Beauclerk; accompanied by notes historical and descriptive, (London, A. Flint pour l'édition originale), Belleville, Mika, 1980. Au moment des insurrections, Beauclerk était lieutenant du Royal Regiment of Foot sous le commandement du colonel Wetherall. Il avait obtenu ce grade en 1836, juste avant sa participation aux campagnes de Saint-Charles et de Saint-Eustache à l'automne 1837. À son retour en Angleterre, en 1839, il obtint celui de capitaine. Mentionnons également que Beauclerk est le fils du $8^{e}$ Duc de St. Albans. Voir John Russell Harper, Early Painters and Engravers in Canada, Toronto, University of Toronto Press, 1970, p. 21.

${ }^{55}$ Voir l'exposition virtuelle The Illustrating Traveller: Adventure and illustration in North America and the Caribeean 1760-1895, préparée par William S. Reese et George Miles, sur le site de Yale University Beinecke Rare Book \& Manuscript Library Exhibition, section "Valor and Endurance, Part I of III ", dernière révision le 4 septembre 1996, http://www.library.yale.edu/beinecke/valor1.htm, consulté le 31 janvier 2008.
} 
Wetherall Advancing to the Capture of St. Charles, 25th November 1837; Attack on St. Charles, 25 th November, 1837; Front View of the Church of St. Eustache, Occupied by the Insurgents - the Artillery Forcing an Entrance, 14th December, 1837 et Back View of the Church of St. Eustache and Dispersion of the Insurgents, $14^{\text {th }}$ December, 18370), toutes influencées par les règles du picturesque ${ }^{57}$. Cette approche esthétique était à mi-chemin entre la conception du beau et celle du sublime ou, si l'on veut simplifier, entre une représentation de l'idéal et celle qui transcende cet idéal. Il est aussi accepté que le sublime, grand et terrifiant, s'oppose au beau harmonieux. Toutefois, qu'il s'agisse du beau ou du sublime, et dans ce cas-ci du picturesque, ces théories rejettent totalement l'idée de représenter le réel tel qu'on le conçoit, c'est-à-dire conforme à ce qui a été vu ou vécu. C'est d'ailleurs ce qui explique les inexactitudes, tant des faits dépeints que de l'espace représenté, constatées par certains médiateurs de la mise en mémoire des patriotes.

En fait, en privilégiant une composition formelle qui met l'accent sur le lieu où se déroule l'action et en magnifiant les interventions militaires des soldats de Sa Majesté à l'endroit des patriotes, Beauclerk s'est inscrit non seulement dans une pratique esthétique familière dans l'Angleterre des XVIII et $\mathrm{XXX}^{e}$ siècles, mais il a également apporté une contribution importante à l'énonciation d'un art national ${ }^{58}$ voire, pour reprendre

${ }^{56}$ Estampes, encre et aquarelle sur papier (lithographiées par Nathaniel Hartnell selon les esquisses originales de Beauclerk), $26,5 \mathrm{~cm}$ x 36,6 cm. Tirées à plusieurs exemplaires, on les trouve dans plusieurs collections muséales et archivistiques dont le Musée McCord, Musée du Château Ramezay, Musée de la civilisation (bibliothèque du séminaire), Bibliothèque et Archives Canada (Collection Peter Winkworth et Collection W.H. Coverdale) et Bibliothèque et Archives nationales du Québec (Collection initiale).

57 Règles édictées par William Gilpin dans son traité de peinture, An Essay on Prints, paru en 1768.

${ }^{58}$ Voir Kamille T.H. Parkinson, " Philip John Bainbrigge and the Group of 1838: Imperial Landscapes and The Colonial Art Scene in Canada ", thèse de doctorat (art), Queen's University, 2005, p. 101-108, dans lequel les ouvrages de Kay Dian Kriz, The Idea of the English Landscape Painter: Genius as Alibi in the Early Nineteenth Century, New Haven et Londres, Yale University Press, 1997, p. 7679 et de Greg Smith, Emergence of the Professional Watercolourist: Contentions and 
les mots de Todd Portefield, d'une "imperial culture" ". Plus qu'un document informatif sur la "difficult or dangerous imperial enterprise $^{60}$ » à laquelle il avait participé, les lithographies tirées des dessins de Beauclerk affirmaient la grandeur et la puissance de l'Empire britannique sur ses colonies. On peut même supposer que la publication de ces Lithographic Views, dans la foulée du dépôt du rapport Durham, justifiait les opérations menées à Saint-Charles «[t]o dislodge the rebels from [...] their strongholds ${ }^{61}$ » et celles déployées à SaintEustache afin de soumettre ses habitants, comme ceux des villages voisins, " to the will of government ${ }^{62}$ ".

Bien que cette analyse ne repose que sur quelques-unes des œuvres produites à cette époque, il est clair que ces témoignages visuels offrent à voir des perspectives fort différentes. Les regards posés par Lord Charles Beauclerk et Katherine Jane Ellice, tous deux Britanniques, sur les patriotes sont d'ailleurs exemplaires de cette divergence de points de vue, ne serait-ce que sur l'espace qu'ils leur réservent dans leurs œuvres: petits dans celles du militaire, occupant toute l'aquarelle pour la belle-fille du seigneur de Beauharnois. Sous le pinceau du premier, les patriotes ne sont que des insurgés trop faibles pour résister à l'armée britannique, alors que sous celui de la seconde, ils représentent la terreur. Certes, observées sous la loupe attentive du spécialiste des rébellions, les Lithographic Views ne sont pas du tout conforme aux faits colligés. De la même manière, ces mêmes patriotes dépeints dans The Insurgent, At Beauharnois, Lower Canada (Quebec) sont sans doute les mêmes qui furent incarcérés à la prison commune de Montréal, certains d'entre eux ont peut-être été exilés en Australie ou même pendus. Ce sont aussi ces mêmes

Alliances in the Artistic Domain, 1760-1824, Hants, Ashgate Publishing Limited, 2002, p. 26-44 sont largement cités.

59 Todd Porterfield, The Allure of Empire Art in the Service of French Imperialism 1798-1836, Princeton, Princeton University Press, 1998, p. 4.

${ }^{60}$ Voir l'exposition virtuelle The Illustrating Traveller.

${ }^{61}$ Beauclerk, "Preface", Lithographic Views, p. 2.

${ }^{62}$ Ibid., p. 13. 
patriotes pour qui des monuments furent élevés. Néanmoins, remises dans leur contexte de production, ces œuvres permettent de s'introduire dans l'imaginaire visuel des britanniques des années 1830 . Un imaginaire marqué autant du souvenir de la Révolution française qu'avide de représentations célébrant la grandeur de l'Empire.

Les œuvres de Robert-Shore-Milnes Bouchette de même que celles de Jean-Joseph Girouard n'ont pas moins d'importance, et ce, même si elles n'ont pas suscité de critique ou de réappropriation. Elles sont de précieux documents qui ont permis de se faire une idée visuelle d'au moins une pièce de la prison et de dresser un portrait des patriotes. À l'instar des œuvres de Beauclerk et d'Ellice, elles sont représentatives de cette génération de Bas-Canadiens qui, dans les premières décennies du $\mathrm{XIX}^{\mathrm{e}}$ siècle, prirent conscience de la place qu'ils occupaient dans la colonie et du constat qu'ils posaient à l'égard des disparités économiques entre l'élite et le peuple.

\section{Interprétations visuelles sur le sujet des rébellions}

Après la publication des Lithographic Views de Charles Beauclerk, il a fallu près de quarante ans avant que ne soient produites de nouvelles interprétations visuelles des rébellions. Il ne s'agissait plus dès lors, comme le sont les œuvres d'Ellice, de Girouard, de Bouchette ou de Beauclerk, de témoignages d'acteurs de l'histoire, mais bien de regards rétrospectifs sur le passé. Parmi ces représentations des insurrections, on compte un grand nombre d'illustrations réalisées pour des ouvrages historiques, des manuels scolaires, des autobiographies, de la fiction littéraire, voire des articles de journaux. C'est le cas notamment de Papineau addressing meeting de Charles William Jefferys. Toutes ont contribué à ancrer dans l'imaginaire collectif une image du Patriote.

Ces images du Patriote n'ont toutefois pas toujours eu la même portée symbolique. Tour à tour, selon l'époque où elles naissent, les œuvres et les illustrations se sont faites l'écho des multiples regards posés sur cette page de l'histoire, que l'on comprenne les rébellions 
comme une lutte « contre l'impérialisme britannique ${ }^{63}$ ", comme un "mouvement populaire mobilisé contre les privilégiés ${ }^{64}$ ", que l'on s'interroge sur la légitimité et l'opportunité de la révolte ${ }^{65}$, du rôle des patriotes dans l'obtention du gouvernement responsable et de l'indépendance du Canada ${ }^{66}$, ou encore qu'on y voit l'affirmation des «droits de la majorité francophone [et catholique] du Bas-Canada ${ }^{67}$ ».

Depuis leur toute première réception, ces œuvres et ces illustrations ont été investies et réinvesties par de nombreux discours idéologiques, changeant parfois complètement le vouloir dire originel qu'on leur reconnaissait à l'époque de leur création. Ainsi Manifestation des Canadiens contre le gouvernement anglais, à Saint-Charles, en 1837 de Charles Alexander symbolise autant la démarche indépendantiste des patriotes que les fondements républicains de leurs revendications ${ }^{68}$. Il en est de même pour Papineau addressing meeting qui sert depuis 2003 à la promotion de la Journée nationale des patriotes. Quant à la maquette pour le monument à Jean-Olivier Chénier, elle est désormais considérée comme une occasion manquée de valoriser le talent d'un artiste canadien-français, voire le symptôme d'un refoulement identitaire ${ }^{69}$. Certes, comme l'a écrit Umberto Eco, l'œuvre reste ouverte à de nombreuses lectures. Néanmoins, si on considère que les représentations des rébellions sont d'abord l'expression de l'époque où elles naissent, il importe de les remettre dans leur contexte de production et de réception et d'interroger les intentions qui leur

\footnotetext{
${ }^{63}$ Bernard, Les rébellions de 1837-1838, p. 7.

${ }^{64}$ Ibid.

${ }^{65}$ Ibid., p. 27.

${ }^{66}$ Ibid., p. 32.

${ }^{67}$ Ibid., p. 7.

${ }^{68}$ Pour son ouvrage Le Printemps de l'Amérique française: américanité, anticolonialisme et républicanisme dans le discours politique québécois, 1805-1837, Montréal, Boréal, 1995, Louis-Georges Harvey a choisi cette œuvre afin d'illustrer la page couverture.

${ }^{69}$ Je fais ici référence à Marc Collin, Autour de Chénier: les rébellions et la conscience historique canadienne et québécoise, thèse de doctorat (histoire), Université Laval, 2006.
} 
sont sous-jacentes, qu'il s'agisse de celles du commanditaire ou de l'artiste.

\section{Manifestation des Canadiens contre le gouvernement anglais, à Saint-Charles, en 1837, 1891}

Aussi connue sous le titre de L'Assemblée des six comtés, Manifestation des Canadiens contre le gouvernement anglais, à Saint-Charles, en $1837 \mathrm{du}$ peintre canadien-anglais Charles Alexander ${ }^{70}$, cette toile est une œuvre majeure de l'iconographie rébellienne. C'est aussi un des rares tableaux d'histoire, de l'ordre de la grande machine - l'œuvre mesure 300 centimètres de haut par 690 centimètres de large -, que compte le corpus d'art canadien. Longtemps demeurée dans l'oubli ${ }^{71}$, elle est accrochée aux cimaises du Musée national des beaux-arts de Québec depuis 1995.

La commande pour un tel tableau d'histoire viendrait, selon toute vraisemblance, de Raymond Préfontaine, représentant d'Hochelaga au Conseil municipal de Montréal et député libéral à la Chambre des communes. Exposée au Palais des Arts et de l'Industrie

${ }^{70}$ Né Charles Alexander Smith, il quitta le Canada vers la fin du XIX ${ }^{e}$ siècle pour Détroit au Michigan puis s'installa définitivement à Londres en 1900. C'est là qu'il décéda en 1915. J. Russell Harper, Early Painters and Engravers in Canada, p. 6.

${ }^{71}$ On connaît bien peu de chose sur cette œuvre. Outre un article de Robert Derome sur les peintures du palais législatif de Québec ("Charles Huot et la peinture d'histoire au Palais législatif de Québec (1883-1930)", Bulletin de la Galerie nationale $d u$ Canada, $\mathrm{n}^{\circ} 27,1976$, p. 2-37) et le catalogue du Musée national des beaux-arts du Québec (Yves Lacasse et John R. Porter, dir., La collection du Musée national des beaux-arts du Québec: une histoire de l'art du Québec, Québec, Musée national des beaux-arts du Québec, 2004, p. 74), bien peu d'historiens de l'art s'y sont intéressés. Les quelques jalons de la vie de l'œuvre méritent toutefois d'être mentionnés. Après le décès d'Honoré Mercier en 1894, sa veuve se serait départi de l'œuvre. En 1930, la province en fait l'acquisition, puis en 1937, année du centenaire des rébellions, le premier ministre du Québec, Maurice Duplessis, transfere l'œuvre au Musée de la Province (actuel MNBAQ) où, semble-t-il, elle aurait été exposée pendant quelques temps. Elle est ensuite oubliée dans les réserves du Musée pendant plus de 50 ans. Elle a finalement été restaurée en 1990, grâce à un don des Amis du MNBAQ, puis accrochée de façon permanente en 1995, année du référendum, dans l'une des salles du MNBAQ consacrées à l'art historique. 
à Paris au printemps 1891, cette représentation de l'Assemblée des comtés de Richelieu, de Saint-Hyacinthe, de Rouville, de Chambly, de Verchères et de l'Acadie, tenue à Saint-Charles (sur Richelieu), le 23 octobre 1837 séduit Honoré Mercier, alors premier ministre du Québec, de passage à Paris. Il s'en porte acquéreur dans le dessein avoué qu'elle orne un mur du Parlement de Québec. Ce projet n’a toutefois pas connu de suite.

Au moment où elle était réalisée, l'œuvre récolta de belles critiques. C'est du moins ce qu'on peut conclure à la lecture des articles colligés dans les différents dossiers d'archives qui ont été consultés. Un premier article parut d'abord à Paris, sous la plume de Maurice O'Reilly dans les pages de Paris-Canada ${ }^{72}$, édition du 27 décembre 1890. Après avoir louangé le peintre et témoigné de toutes les reconnaissances que suscitait son talent, O'Reilly s'attarda sur « la grande œuvre à laquelle Charles Alexander se consacr[ait] ». D'entrée de jeu, le journaliste mentionna qu'il s'agissait d'une commande venant de personnalités du Québec et que l'œuvre qui représentait l'Assemblée des six comtés était "destinée au Parlement de Québec". Suivait une description pour le moins enthousiaste de l'œuvre dans laquelle on peut lire que « les notables qui prirent part à cette assemblée fameuse ", dont les Louis-Joseph Papineau, Wolfred Nelson, JosephToussaint Drolet, Amury Girod, Pierre Boucher-Belleville, Louis Lacoste, le capitaine Joseph Vincent de Longueuil et René Boileau de Chambly, étaient « autant de noms qui [faisaient] battre les poitrines canadiennes». Selon O’Reilly, l'œuvre, synonyme d'« une puissance

\footnotetext{
${ }^{72}$ Maurice O'Reilly, «Nos artistes à Paris», Paris-Canada, 27 décembre 1890. Le journal Paris-Canada avait été fondé par Hector Fabre, premier commissaire général du Canada à Paris entre 1884 et 1910. Le but avoué était de promouvoir les relations franco-canadiennes. Hector Fabre était le fils d'Édouard-Raymond Fabre, libraire patriote, le neveu de Charles-Ovide Perrault, tué lors de la bataille de Saint-Denis en novembre 1837, et de Louis Fabre, propriétaire du Vindicator, le frère d'Édouard-Charles, premier archevêque de Montréal, et d'Hortense, épouse de George-Étienne Cartier. Notons que la majorité des articles de journaux auxquels il est fait référence sont tirés des dossiers d'archives, ce qui explique l'absence de numéro de page et, parfois, de date.
} 
de vie et [d']une fougue d'exécution", ne pouvait qu' "assure[r] à l'artiste un éclatant succès ». D'autant, ajoutait-il, que les « recherches les plus minutieuses, tant sur les costumes de l'époque que sur les personnages historiques » auxquelles s'était livré Alexander, appelaient "un véritable retentissement au Canada ». Cet article fut repris presque mot à mot dans l'édition du 8 janvier 1891 de La Presse et dans l'édition du 22 janvier 1891 du Courrier du Canada. Le 28 mars 1891, Paris-Canada publiait un compte rendu des déplacements du premier ministre Honoré Mercier à Paris ${ }^{73}$. On y rapportait que le plus haut représentant de la province avait vu au Palais de l'Industrie ${ }^{74}$ la toile représentant l'Assemblée des six comtés de Charles Alexander et qu'il avait «vivement félicité" l'artiste. On soulignait aussi que l'artiste avait dépeint un Papineau plein de "noblesse et de grandeur " et que le tableau était "admirablement composé et d'un effet saisissant". Le 8 avril suivant, L'Électeur de Québec rendait publique une lettre d'Honoré Mercier dans laquelle, après avoir donné des détails sur les affaires qu'il était allé régler à Paris, le premier ministre affirmait son enthousiasme à l'égard de cette œuvre représentant «un des plus beaux et des plus glorieux souvenirs de notre histoire nationale", une œuvre qui de surcroît, soulignait-il, avait été « réalisée par un Canadien anglais".

Cette appréciation de Manifestation des Canadiens contre le gouvernement anglais à Saint-Charles, en 1837 de la part du premier ministre du Québec n'est certes pas étrangère au fait que son père était parmi les fidèles de Louis-Joseph Papineau et que lui-même fut «élevé dans la mystique des événements de 1837-1838 ${ }^{75}$ ». Il n'était pas non plus anodin qu'il prît la peine de souligner qu'il reconnaissait « avec plaisir ${ }^{76}$ » et même «émotion, les figures animées et vigoureuses

\footnotetext{
${ }^{73}$ Ce dernier était allé en France afin de négocier un prêt pour consolider les finances publiques du Québec.

${ }^{74}$ Il s'agit du Grand Palais, construit sur les Champs-Élysées à Paris en 1855.

${ }^{75}$ Dictionnaire biographique du Canada en ligne, entrée " Honoré Mercier ", http:// www.biographi.ca, consulté le 4 septembre 2009.

${ }^{76}$ L'Électeur, 8 avril 1891.
} 
de nos bons cultivateurs ${ }^{77}$ ", qui avaient trouvé place, au même titre que les chefs patriotes, dans cette fresque historique. Il était lui-même fils et petit-fils d'agriculteur. Plus qu'une critique esthétique d'une œuvre d'un compatriote de langue anglaise exposée en France, ce commentaire pouvait se lire comme l'affirmation d'une histoire canadienne-française qui serait tout aussi importante que celle des peuples européens. Sans exprimer clairement qu'il destinait le tableau d'Alexander au Parlement de Québec, Mercier s'assurait qu'il soit bien reçu.

L'œuvre n'a toutefois pas trouvé place dans l'enceinte du Parlement. Destitué de son poste de premier ministre en raison du scandale de la Baie-des-Chaleurs, Honoré Mercier fut remplacé en décembre 1891 par le conservateur Charles-Eugène Boucher de Boucherville. Selon Robert Derome, ce dernier jugeait la toile d'Alexander trop "révolutionnaire ${ }^{78}$ " et s'opposa à son accrochage. N'était-ce là qu'une vile querelle entre libéraux et conservateurs? Peut-être.

Toutefois, si on regarde d'un peu plus près ce tableau historique, on remarque que certains motifs iconographiques font moins référençe au Bas-Canada de 1837-1838 et à la ferveur patriote qu'à la France républicaine. C'est le cas notamment du bonnet phrygien qui, en plus d'orner la colonne élevée en l'honneur de Papineau lors de cette fameuse assemblée d'octobre 1837, remplace le bonnet de laine que portaient alors les paysans. Pourtant, ni le compte rendu paru dans le journal La Minerve (9) $^{9}$ dans les jours qui ont suivi l'Assemblée des six comtés, ni le récit Les Patriotes $1837-1838^{80}$ de Laurent-Olivier

77 Ibid.

${ }^{78}$ Derome, "Charles Huot et la peinture d'histoire au Palais législatif de Québec (1883-1930)", p. 7.

79 "Grande Assemblée de la Confédération des Six Comtés ", La Minerve, 30 octobre 1837 , p. 2.

${ }^{80}$ Laurent-Olivier David, Les patriotes de 1837-1838, Montréal, Beauchemin, 1937, p. 33. Il s'agit d'une réédition de l'ouvrage paru chez Eusèbe Senécal \& Fils en 1884. 
David publié en 1884, ni cet autre texte paru dans les pages du Montreal Star en $1887^{81}$ ne font allusion à un tel accessoire vestimentaire qui aurait été porté par les patriotes. Les seules mentions à propos de l'habillement sont à l'effet que "Papineau ${ }^{82}$ " et «l'élite des Patriotes ${ }^{83}$ " portaient l'étoffe du pays. Cette interprétation d'Alexander est pour le moins surprenante, d'autant que Maurice O'Reilly, dans les pages du Paris-Canada de décembre 1890, affirmait, je le rappelle, que l'artiste avait effectué des " recherches minutieuses ${ }^{84}$ " sur les costumes de l'époque.

Le motif de la colonne pose aussi problème. Inspirés sans doute de la tradition de l'arbre de mai ${ }^{85}$, les habitants de Saint-Charles avaient érigé, lors de la tenue de l'Assemblée des six comtés, "une superbe colonne surmontée du "bonnet de la liberté ${ }^{86 "}[\ldots]$ en l'honneur de Papineau ${ }^{87}$ " avec l'inscription suivante: «À Papineau, ses compatriotes reconnaissants, $1837^{88} »$. Il s'agissait vraisemblablement d'un "poteau de la liberte ${ }^{89}$ » ou encore d' « un grand

${ }^{81}$ «1837-1838 - La Grande Insurrection! The Great Insurrection!» The Montreal Star, 28 septembre 1887, p. 3.

82 "Grande Assemblée de la Confédération des Six Comtés », La Minerve, 30 octobre 1837, p. 2.

${ }^{83}$ David, Les patriotes de 1837-1838, p. 33.

${ }^{84}$ Maurice O’Reilly, "Nos artistes à Paris", Paris-Canada, 27 décembre 1890.

${ }^{85}$ Une tradition qui, depuis l'époque de la Nouvelle-France jusqu'au milieu du $\mathrm{xIx}^{e}$ siècle, voulait que le premier jour de mai, les miliciens plantent un arbre devant la maison de leur capitaine de milice en signe de fidélité. Voir «Le caractère de la milice ", Patrimoine militaire canadien, t. I, Annexe B, La vie quotidienne en Nouvelle-France, La Milice, http://phmc.gc.ca/cmh/fr/page_213.asp, consultéle 7 avril 2006. Cette tradition était aussi partagée par les habitants qui, le $1^{\text {er }}$ mai, plantaient un arbre ébranché devant le manoir seigneurial, le noircissant à coup de fusil devant leur seigneur.

${ }^{86}$ Ici l'énoncé "bonnet de la liberté" réfère non pas au bonnet phrygien mais plutôt au fait que les habitants portaient le bonnet fait de la laine des moutons du pays. Il faut aussi préciser que le bonnet était généralement bleu et non rouge. Voir Francis Back, "Le bonnet bleu des patriotes», Cap-aux-diamants, $\mathrm{n}^{\circ}$ 61, (printemps 2000), p. 2.

87 "Grande Assemblée de la Confédération des Six Comtés ", La Minerve, 30 octobre 1837, p. 2

${ }^{88} \mathrm{Ibid}$.

${ }^{89}$ Ibid. 
mai ${ }^{90}$ " semblable à celui reconstitué à Saint-Charles en novembre 1982, semblable aussi au dessin qu'en fit Henri Julien pour l'édition du 28 septembre 1887 du Montreal Star. Pourtant, la colonne peinte par Alexander évoque beaucoup plus la Colonne de Juillet élevée sur la place de la Bastille à Paris afin de commémorer la Révolution française et, plus particulièrement, la prise de la Bastille du 14 juillet 1789, que le simple hommage rendu au leader patriote par ses concitoyens. D'ailleurs, il est fort peu probable qu'à cette époque les patriotes aient eu les moyens financiers de se payer un tel ornement. Ce choix de l'artiste est d'autant plus discutable que la symbolique liée à la colonne, corinthienne de surcroît, n'est pas de l'ordre de l'honneur rendu - la signification courante de l'arbre de mai - mais bien plutôt de celui de la victoire. Or, faut-il le rappeler, à l'automne 1837, les patriotes n'avaient rien gagné!

Certes, le fait qu'Alexander vivait à Paris ${ }^{91}$ au moment de la réalisation de Manifestation des Canadiens contre le gouvernement anglais à Saint-Charles, en 1837, sa connaissance des œuvres françaises et sa fréquentation des ateliers d'artistes ont vraisemblablement influencé ses choix esthétiques. Le traitement de certains personnages à l'avant-plan du tableau, qui rappellent les scènes de cafés dépeintes par les impressionnistes ${ }^{92}$, en est un exemple. Néanmoins, il importe de mentionner que de ce côté-ci de l'Atlantique, encore en 1891, les rébellions soulevaient les passions. Trois ans à peine avant qu'Alexander entreprenne la réalisation de Manifestation des Canadiens contre le gouvernement anglais à Saint-Charles, en 1837, le Montreal Star avait publié sa série 1837-1838 La Grande Insurrection! The Great Insurrection! dans laquelle la parole avait été largement laissée à ceux

${ }^{90}$ "1837-1838 - La Grande Insurrection! The Great Insurrection!», The Montreal Star, 28 septembre 1887 , p. 3.

${ }^{91}$ À cette époque, bon nombre d'artistes canadiens séjournaient en France afin de parfaire leur formation. Alexander lui-même a fréquenté l'atelier des Lefebvre et Boulanger ainsi que l'Académie Julian à Paris.

92 Je tiens à remercier ici Mario Béland, conservateur au Musée national des beauxarts de Québec, qui, lors de mes recherches sur cette œuvre en janvier 2006, attira mon attention sur ce détail esthétique. 
qui matèrent le mouvement patriote, qu'il s'agisse de volontaires, de membres de l'armée ou encore du clergé. Même les témoignages issus de l'expérience patriote "dénon[çai]ent, pour reprendre les mots de Jean Saint-Onge, le fait qu'on puisse prendre les armes pour contester l'autorité ${ }^{3}$ " en place. À propos de l'assemblée qui eut lieu à SaintCharles, le Montreal Star avait mis non seulement l'accent sur l'appel aux armes prononcé par Nelson et sur la désobéissance civile inscrite dans les treize résolutions adoptées par les représentants des six comtés, mais faisait également un parallèle entre les rébellions et la Révolution française. On rappelait notamment que cette promesse de fidélité déclarée à Papineau devant la colonne était du "genre de celles si fréquentes lors de la grande révolution de $89^{94}$ ".

Est-il alors possible de penser que la présence de bonnets phrygiens ou d'une possible réplique de la Colonne de Juillet serait à l'origine du refus de Boucher de Boucherville d'exaucer le vou de Mercier? Est-ce à ces symboles de la Révolution française que faisait référence Robert Derome, lorsqu'il affirmait que Boucher de Boucherville trouvait l'œuvre trop "révolutionnaire"? Si, comme je le soutiens, une œuvre, quel que soit le sujet peint, est emblématique de la période qui la voit naître, on peut certainement le penser.

En 1890, lorsque Charles Alexander reçoit de Raymond Préfontaine la commande d'un tableau d'histoire, le Québec, par l'entremise d'Hector Fabre, tissait des liens de plus en plus serrés avec la France. Cette relation se vivait toutefois à l'heure de la III ${ }^{e}$ république et elle n'était pas toujours bien vue, ni par l'Angleterre, ni par les conservateurs et les ultramontains, pour qui la seule France susceptible d'être aimée

${ }^{93}$ Jean Saint-Onge, "Une idée de la subversion dans les gravures de Henri Julien », mémoire de maîtrise, Université Laval, 1977, cité dans Claude Cossette, Les images démaquillées ou L'iconique: comment lire et écrire des images fonctionnelles pour l'enseignement, le journalisme et la publicité. Québec, Éditions Riguil internationales, 1982, p. 48-64, http://www.comviz.com.ulaval.ca/module1/1.4_mainenmain.php, consulté le 6 mars 2005.

94 «1837-1838 - La Grande Insurrection! The Great Insurrection!», The Montreal Star, 28 septembre 1887, p. 3. 
était, selon les mots de Jules-Paul Tardivel: " la France d'autrefois, la France puissante, grande et glorieuse, la France fille aînée de l'Église ${ }^{95}$ ", bref la France d'avant la Révolution de 1789. Dans un tel contexte, on peut comprendre que les symboles de la Révolution française, peints par Alexander, aient condamné l'œuvre au " purgatoire ${ }^{96}$ "!

\section{Maquette du monument à Jean-Olivier Chénier, 1894}

À la même époque où fut peinte Manifestation des Canadiens contre le gouvernement anglais, à Saint-Charles, en 1837, le docteur Marcil, de Saint-Eustache, entreprit des démarches afin d'exhumer les restes du patriote Jean-Olivier Chénier ${ }^{97}$ et de les translater au cimetière Notre-Dame-des-Neiges pour les inhumer sous le monument aux victimes politiques de 1837-1838, érigé en 1858. Bien qu'il ait obtenu les autorisations nécessaires de l'abbé Maréchal, grand vicaire du diocèse de Montréal, le retour au pays de $\mathrm{M}^{\mathrm{gr}}$ Édouard-Charles Fabre $^{98}$ compromit le projet, celui-ci s'opposant farouchement à l'idée de pardonner aux patriotes morts les armes à la main. Loin d'être abandonnée, l'idée de rendre hommage à Jean-Olivier Chénier refit surface en $1893^{99}$.

${ }^{95}$ Cité dans Yvan Lamonde, Histoire sociale des idées au Québec (1760-1896), Montréal, Fides, 2000, p. 458.

${ }^{96}$ Yves Lacasse et John R. Porter, La collection du Musée national des beaux-arts du Québec, p. 74.

${ }^{97}$ Le corps de Chénier aurait été enterré dans l'ancien cimetière destiné aux enfants morts sans baptême à Saint-Eustache, "La démonstration de dimanche ", $L a$ Patrie, 19 juin 1891 .

${ }^{98} \mathrm{M}^{\mathrm{gr}}$ Fabre avait fait un séjour de trois mois au collège canadien des sulpiciens, à Rome. Dictionnaire biographique du Canada en ligne, http://www.biographi.ca/, consulté le 13 novembre 2007. Lire aussi Tancrède Marcil, «Où sont les restes de Chénier?", Le Jour, 30 octobre 1937, p. 8.

99 Selon Pierre-Georges Roy, l'idée d'élever un monument à Jean-Olivier Chénier remonterait à 1887. À la suite du refus de l'Église de translater les restes de Chénier sous le monument aux Victimes politiques de 1837-1838, le Dr Marcil tenta, encore sans succès, de faire élever un monument à celui qui avait "bravement " mené la bataille du 14 décembre 1837. Pierre-Georges Roy, Les monuments commémoratifs de la province de Québec, Québec, Commission des monuments historiques de la province de Québec, 1923, p. 245. 
Un an plus tard, les médiateurs de ce geste mémoriel, qui comptaient dans leurs rangs. Honoré Mercier et Louis Fréchette à la vice-présidence d'honneur ${ }^{100}$, présentaient au grand public une maquette du monument réalisée par le sculpteur Louis-Philippe Hébert (figure 1) ${ }^{101}$. Cette dernière représentait « un des plus braves enfants du Canada français ${ }^{102}$ ", un "Chénier en capot d'étoffe du pays, en bottes molles, la ceinture autour du corps, tête nue, un vieux mousquet à la main et tendant le bras vers l'ennemi ${ }^{103}$ ". Sur le socle du monument, on devait y graver «le dernier cri de Chénier: Vive la Liberté ${ }^{104}$ !» Dix jours plus tard, Le Monde Illustré publia une reproduction de la maquette et dépeignit l'œuvre en ces termes:

L'attitude du héros de Saint-Eustache est vraiment superbe. Tout frémissant, les cheveux au vent, il serre encore dans sa main le pauvre et rustique fusil qui bientôt deviendra inutile! Sans s'occuper du boulet anglais qui vient de trouer la terre sous ses pieds, il regarde au loin le champ de bataille où va mourir cette liberté pour laquelle il a tant combattu!

Une expression à la fois douloureuse et énergique est peinte sur son visage: tout est perdu, la victoire s'est envolée, et les lèvres du héros s'entrouvrent déjà, dans son désespoir pour lancer ce cri sublime de: «Vive la liberté!” que ses concitoyens ont pieusement conservé dans leur cœur, en attendant qu'ils le gravent en lettres inneffaçables $[s i c]$ sur le piedestal $[s i c]$ du monument ${ }^{105}$.

${ }^{100}$ Le comité était formé du docteur David Marcil, président d'honneur, d'Honoré Mercier, de Louis Fréchette et de Joseph-Maxime Beausoleil, vice-présidents d'honneur, de L. Forget et de Joseph-Octave Pelland, secrétaires, de L. J. Hérard, trésorier, et de Georges Alphonse Dumont, sous-trésorier, ainsi que de P.-E. Paquette, de J.-G. Héroux, de L.-J. Lamontagne et d'[Alfred] Savard. Le Monde Illustré, vol. II, n 545 (13 octobre 1894), p. 282-283.

${ }^{101}$ Hébert a été formé par Napoléon Bourassa et est reconnu pour être l'un des meilleurs sculpteurs canadiens.

102 "Chénier ", La Patrie, 26 août 1895, p. 1.

103 "Le Monument Chénier", La Patrie, 3 février 1894.

${ }^{104}$ Il faut noter ici que ce cri de "Vive la liberté! " est plutôt celui de Charles Hindelang lorsqu'il fut pendu et non de Chénier. Thomas Chevalier DeLorimier a aussi écrit ces mots en conclusion de ce que l'on considère comme son testament politique.

105 «Monument Chénier », Le Monde Illustré, vol. 10 (24 février 1894), p. 507. 
La campagne de souscription qui suivit ne suscita pas l'enthousiasme espéré. En octobre 1894, huit mois après la présentation de la maquette d'Hébert, un appel au patriotisme était donc lancé dans Le Monde Illustré:

C'est à Montréal, sur l'une des principales places publiques, que sera élevée la statue du grand patriote, qui n’a pas craint de sacrifier sa vie afin de donner à son pays les libertés dont il jouit aujourd'hui.

La pensée patriotique qui anima ces hommes mérite l'approbation de tous ceux qui cultivent le culte des héros morts pour la patrie. Et Le Monde Illustré comme toujours, est heureux de venir offrir son tribut d'hommage à ses citoyens reconnaissants.

Nous espérons que le peuple de cette province, sans aucune distinction de race ni de parti, se fera un devoir de venir seconder les efforts de messieurs les membres du comité Chénier, afin de lui permettre de réaliser au plus tôt le beau projet qu'ils ont formé ${ }^{106}$.

Malgré cet appel, le comité ne réussit pas à recueillir les $5000 \$$ qu'aurait coûté la réalisation de la maquette d'Hébert ${ }^{107}$. Le jour même de l'inauguration, le total des contributions s'élevait tout juste à 1242,69 \$108.

Certes, les divergences idéologiques quant à la place que devaient occuper les patriotes dans la mémoire collective constituent une part importante de la controverse associée à cette démarche commémorative. Dans Making Public Pasts: The Contested Terrain of Montreal's Public Memories, 1891-1930109, Alan Gordon soutient d'ailleurs que la polémique entourant ce geste tenait essentiellement

106 "Monument Chénier ", Le Monde Illustré, vol. 11, nº 545 (13 octobre 1894), p. 279.

${ }^{107}$ Correspondance personnelle de Philippe Hébert, Musée national des beaux-arts de Québec, cité dans Collin, "Autour de Chénier ", p. 1.

${ }^{108}$ Alan Gordon, Making Public Pasts: The Contested Terrain of Montreal's Public Memories, 1891-1930, Montréal et Kingston, McGill-Queen's University Press, 2001, p. 8. Le monument inauguré le 27 août 1895 en bordure de l'ancien square Viger est l'œuvre du sculpteur Alfonzo Pelzer de la firme W.-H. Mullins (Salem, Ohio).

${ }^{109}$ Ibid., p. XI-XVI. 
des positions contradictoires qu'entretenaient les libéraux et l'Église à l'égard des rébellions. Les premiers y voyaient le processus d'obtention des libertés politiques, alors que la seconde pouvait difficilement ou ne voulait pas revenir sur l'opinion (et les excommunications) qu'elle avait émises à l'époque des insurrections. Il faut aussi rappeler que l'opposition à la mise en mémoire des rébellions venait aussi de certains citoyens de Saint-Eustache, particulièrement des descendants de ceux qui s'étaient opposés aux patriotes en 1837 et dont le plus connu est certainement Charles-Auguste-Maximilien Globensky ${ }^{110}$. Certains discours tenus lors de l'inauguration du monument ou comptes rendus parus au lendemain de cette dernière sont significatifs du débat idéologique qui entoura la médiation mémorielle de JeanOlivier Chénier. À titre d'exemple, dans La Patrie, journal lié au Parti libéral, on mentionnait qu' " [e]n dépit d'une température affreuse et des appels aux préjugés et au fanatisme, trois mille personnes au moins s[étaient] rendues à l'inauguration du monument élevé à la mémoire de ce héros tombé au champ d'honneur, en combattant pour la liberté ${ }^{111} »$. Défiant sans doute l'opposition qui était relayée dans la presse tant anglophone que francophone, on ajoutait: «N'estce pas là une réponse publique et solennelle aux détracteurs de la mémoire du héros de St-Eustache [sic] ${ }^{112}$ ? ” De son côté, le journal La Minerve, d'allégeance conservatrice et opposé au projet, soutenait que «le but poursuivi [par les médiateurs de la mise en mémoire] n'était pas tant d'honorer la mémoire de Chénier que de se venger du clergé ${ }^{113}$ ». Pour ce journal, le monument n'était qu' «une provocation inopportune ${ }^{114} \%$.

\footnotetext{
${ }^{110}$ Ce dernier avait fait paraître, une dizaine d'années auparavant, La Rébellion de 1837 à Saint-Eustache dans lequel il défendait la réputation de son père Maximilien Globensky, commandant des volontaires lors de la bataille de Saint-Eustache. 111 "Chénier Le Dévoilement de la Statue " La Patrie, 26 août 1895.

112 Ibid.

113 «Le Monument Chénier», La Minerve, 26 août 1895.

${ }^{114}$ Ibid.
} 


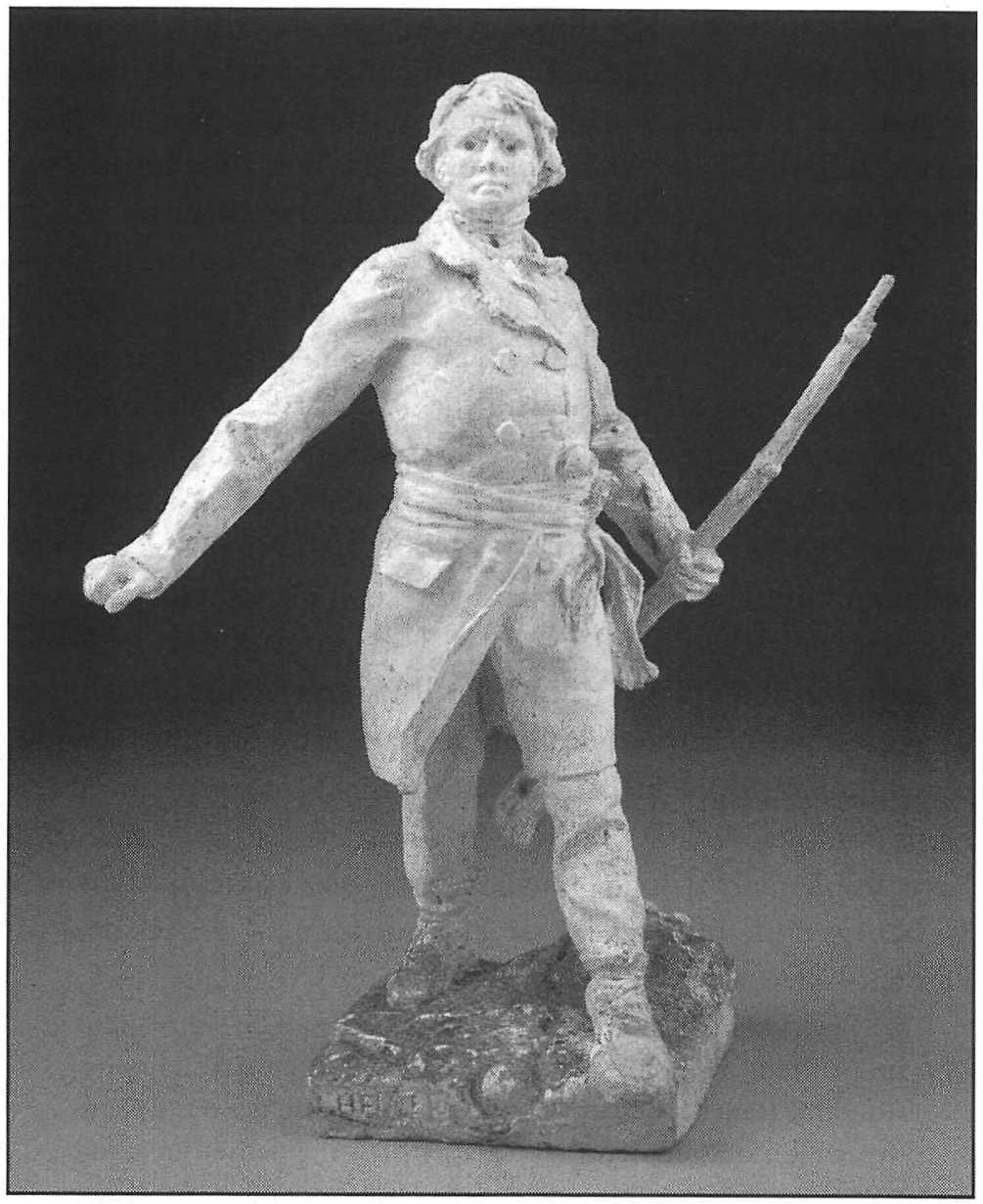

Figure 1- Louis-Philippe Hébert, Maquette pour le "Monument à Jean-Olivier Chénier ", 1894, plâtre, $32 \mathrm{~cm}$ (haut), Musée des beaux-arts de Montréal (Achat legs Horsely et Annie Townsend; n 1984.27; photo: Christine Guest, MBAM). 
Néanmoins, au-delà du geste lui-même de mise en mémoire des patriotes à une époque où l'on questionnait toujours «la légitimité de la révolte et celle de son opportunité ${ }^{115}$ ", il est possible que l'image même de Jean-Olivier Chénier proposée par Louis-Philippe Hébert pour le monument fut un élément important de cette polémique.

Lorsque Louis-Philippe Hébert présenta sa maquette, la statutaire commémorative, ici comme ailleurs, empruntait généralement au style académique européen. Personnages plus grands que nature, attributs de gloire, attitudes solennelles et poses héroïques étaient au nombre des caractéristiques que reproduisaient les sculpteurs afin de répondre au goût des commanditaires. Bref, il s'agissait d'une représentation idéalisée d'un personnage ou d'un événement que l'on voulait immortaliser dans la pierre. Or, si on regarde attentivement le Chénier d'Hébert (ou si on relit le descriptif paru dans Le Monde illustré), on note qu'il n'a ni une attitude solennelle, ni une pose héroïque. Le seul attribut de gloire est un vieux mousquet. Le mouvement qu'imprègne Hébert au personnage (les cheveux au vent, le corps contorsionné, la position des bras, les plis du vêtement) et l'expression qu'il lui donne (visage résigné, point serré) sont un écho au panégyrique qu'avait brossé une quinzaine d'années plus tôt Laurent-Olivier David:

[...] peu grand, mais robuste, les épaules larges, la tête imposante, un peu renversée en arrière, les membres musculeux, une physionomie franche, ouverte, le regard fier et hardi, des traits pleins de virilité, des manières vives, la parole véhémente, un esprit prompt et logique, une âme enthousiaste, faite pour le sacrifice et le dévouement. [Bref, u]ne figure de maréchal de France, une nature de soldat ${ }^{116}$.

Ou encore, à la description livrée par Charles-Auguste-Maximilien Globensky dans son ouvrage sur la rébellion de Saint-Eustache:

[Il] était d'une stature moyenne, trapue et non élégante. L'expression des traits du visage annonçait la vivacité, la ténacité et le désir

${ }^{115}$ Bernard, Les Rébellions de 1837-1838, p. 27 et suivantes.

${ }^{116}$ Laurent-Olivier David, "Les Hommes de 37-38 - Dr. Chénier ", L'Opinion publique, 22 février 1877, p. 88. 
marqué de commander, de triompher en tout et partout. Le coloris du visage comme le teint de sa barbe et de sa chevelure avait une nuance très prononcée sur le roux. [...].

D'un tempéramment $[$ sic $]$ sombre et violent, il attaquait avec impétuosité tous ceux qui ne partageaient pas ses opinions personnelles et politiques. Il essayait à exciter, à ameuter, sans raison, les habitants de St. Eustache [sic] contre tous les notables et les loyaux qui formaient la très grande majorité de l'endroit [...]. Hardi, dévoré par l'ambition de se placer au premier rang, il déployait son activité et tous ses pouvoirs d'intrigue pour aigrir, pour soulever le peuple et le forcer à l'accepter comme chef. [...] Jalousant les autres chefs de la révolte, il ne leur cédait point le pas et il devait devenir insensiblement l'un des hommes les plus en évidence parmi les meneurs de l'insurrection ${ }^{117}$.

En reproduisant ainsi un portrait réel du personnage, ou du moins qui apparaissait comme tel, Hébert était loin de suivre les enseignements de David d'Angers ${ }^{118}$ pour qui le moment représentatif de la vie du personnage à immortaliser devait être celui transcrit dans la statuaire. Son Chénier n'avait pas «l'attitude grave et imposante qu'il devait avoir au moment d'engager l'action qui devait lui coûter la vie ${ }^{119}$ ». En fait, les libertés formelles que s'est permises Hébert relèvent plus d'une esthétique impressionniste - l'impressionnisme étant essentiellement un art pictural, j'entends ici par esthétique impressionniste une traduction des mouvements et de la vie, aussi fugace soit-elle - que du style académique plus conservateur. Comme la plupart des comités ou jurys engagés dans le processus de commande

${ }^{117}$ Charles-Auguste-Maximilien Globensky, La Rébellion de 1837 à Saint-Eustache, précédé d'un exposé de la situation politique du Bas-Canada depuis la cession, Québec, Imprimerie A. Coté, 1883, p. 316-317.

${ }^{118}$ Corinne Prével-Montagne affirme dans sa thèse de doctorat que David d'Angers (1788-1856) aurait, à travers sa pratique, établi une codification de la statutaire publique, notamment pour le style didactique des œuvres commémoratives. La représentation des grands hommes dans la sculpture publique commémorative en Bretagne 1685-1945. Les pratiques, les sculpteurs et les æuvres, thèse de doctorat, Université de Rennes II Haute Bretagne, 2003.

119 "La Statue Chénier", Le Monde Illustré, vol. 12, n 592 (7 septembre 1895), p. 267. 
d'une œuvre commémorative, celui chargé de la matérialisation du projet de monument à la mémoire de Chénier n'avait que peu ou pas de connaissances du domaine de l'art. Il est donc possible qu'il préféra, compte tenu de la controverse entourant cette médiation mémorielle et également en raison du faible nombre de souscriptions, opter pour une sculpture qui s'inscrivait dans les goûts de l'époque plutôt que pour une œuvre dont les qualités formelles détonnaient par rapport à l'ensemble du patrimoine commémoratif déjà érigé.

\section{Papineau addressing meeting, 1912}

La dernière œuvre qui fait l'objet de cette étude est Papinean addressing meeting ${ }^{120}$ du peintre Charles William Jefferys ${ }^{121}$, une aquarelle et crayon sur planche commerciale faisant $25,8 \mathrm{~cm}$ de large sur 38,2 cm de haut. Elle a vraisemblablement été créée vers 1912 à la demande de George Nathaniel Morang, éditeur de la série Makers of Canada, afin d'illustrer Papineau Cartier ${ }^{122}$, une double biographie signée Alfred Duclos DeCelles ${ }^{123}$. Elle est désormais conservée à Bibliothèque et Archives Canada.

Comme son titre le laisse sous-entendre, elle représente le leader patriote discourant devant une foule à proximité d'une église, comme

${ }^{120}$ Bien que l'on constate que sur la fiche descriptive de l'œuvre de Bibliothèque et Archives Canada, de même que dans l'ouvrage de Malcolm G. Parks, Les Rébellions de 1837 dans le Haut et le Bas-Canada, album numéro III, dessins de Charles W. Jefferys, s.l., Publication de l'Imperial Oil Limited, s.d., planche 6, l'œuvre soit désignée sous les titres Papineau Addressing a Crowd et Papineau s'adressant à ses partisans, il paraissait plus approprié de respecter le titre donné par l'artiste. Voir "Notes \& Notebooks: Picture Gallery Vol. II (1-19)", Bibliothèque et Archives Canada, Fonds Charles William Jefferys, MG30 D217 - Vol. I.

${ }^{12.1}$ D'origine britannique, Jefferys a immigré au Canada dans sa tendre jeunesse après un court séjour aux États-Unis. Il a passé une bonne partie de sa vie à Toronto. C'est d'ailleurs à çet endroit qu'il décéda en 1951.

${ }^{122}$ L'édition dont il est question ici est la première édition illustrée de Papineau Cartier. L'édition originale est parue quant à elle en 1904.

${ }^{123}$ Alfred Duclos DeCelles était journaliste, écrivain et avocat. Au moment où il rédigea Papineau Cartier pour la série Makers of Canada il occupait le poste de bibliothécaire en chef de la Bibliothèque du Parlement d'Ottawa. 
cela s'est produit à plusieurs reprises au Bas-Canada à la veille des insurrections de 1837. Suivant de près la présentation qu'avait faite Duclos DeCelles, Jefferys a su rendre avec conviction les qualités oratoires du tribun de même que la ferveur de la population venue l'écouter. Toutefois, le rendu pictural de l'assemblée offre quelques divergences quant à la description écrite des assemblées.

D'une part, bien qu'il soit fait référence dans le texte de Duclos DeCelles à l'assemblée tenue à Saint-Laurent en mai 1837 et à l'assemblée regroupant les six comtés à Saint-Charles en octobre de la même année, Jefferys a choisi de camper ce rassemblement patriote devant l'église du village de Saint-Antoine-sur-Richelieu. Un choix intéressant pour ne pas dire intriguant puisqu'en dépit du fait que nombre de citoyens de Saint-Antoine s'impliquèrent dans le mouvement patriote, certains participant même à la bataille de Saint-Denis le 23 novembre 1837, aucune assemblée n'eut lieu dans cette paroisse.

D'autre part, ajoutant à la vraisemblance de la scène, Jefferys reproduisit le drapeau patriote ainsi que quelques-unes des devises brandies lors de ces assemblées. Parmi les plus lisibles, il y a « Papineau et le système électif", "Nos amis du Haut-Canada", "Papineau Indépendance" et "Les 92 résolutions». Encore une fois, Jefferys a pris quelques libertés en regard du texte de Duclos DeCelle puisque ce dernier n'a fait mention que de trois des nombreuses devises rapportées dans les comptes rendus de La Minerve et du Vindicator, les trois premières. De plus, il importe de rappeler qu'au moment où se sont tenues les assemblées qu'évoque Papineau addressing meeting, "les 92 résolutions", formulées trois ans plus tôt, faisaient déjà partie du passé historique des revendications patriotes. En fait, les assemblées populaires tenues entre mai et octobre 1837 avaient justement été mobilisées afin de dénoncer les résolutions Russell adoptées par le gouvernement britannique en mars 1837 , une réponse somme toute négative de l'Angleterre à ces mêmes 92 résolutions. Détail négligeable, mais tout autant significatif de la volonté de Jefferys de se démarquer du texte du Duclos DeCelles, les devises furent reproduites en 
français dans l'aquarelle alors qu'elles avaient été traduites pour Papineau Cartier.

Certes, comme l'affirmait Jefferys, "[a]t its best, and, after, the work of the historical reconstructor is only an approximation ${ }^{124}$ ». Pourtant ce peintre, historien de l'art et auteur d'ouvrages historiques avait le souci de l'exactitude et de la vraisemblance, effectuant lui-même des recherches sur les objets du passé et sur l'histoire. On peut d'ailleurs penser que c'est sur ce souci d'exactitude que repose son choix de peindre l'église de Saint-Antoine plutôt que celle de la paroisse de Saint-Laurent ou encore celle de Saint-Charles. Il connaissait bien cet édifice pour l'avoir visité quelques années auparavant et en avoir fait des croquis ${ }^{125}$.

Mais au-delà de ces possibles explications, il importe de mentionner que Charles William Jefferys est de ceux qui, en plus d'ouvrir la voie à un art typiquement canadien ${ }^{126}$, apporta une importante contribution dans la constitution de l'histoire visuelle du Canada. Il était d'ailleurs convaincu que ses œuvres contribueraient à lá construction d'une identité nationale ${ }^{127}$. En cela, il rejoignait Morang pour qui la série Makers of Canada était «an exercice in nation-building ${ }^{128} »$.

${ }^{124}$ Charles W. Jefferys, "The Visual Reconstruction of History", The Canadian Historical Review, vol. XVII, n 3 (septembre 1936), p. 265.

125 À l'automne 1900, Jefferys avait fait une excursion dans la région du Saint-Laurent au cours de laquelle il s'était arrêté à Saint-Antoine-sur-Richelieu avant de traverser sur l'autre rive (Saint-Denis-sur-Richelieu). Il avait fait des croquis de l'église. Voir Robert Stacey, C.W. Jefferys, Ottawa, Musée des beaux-arts du Canada, 1985, p. 16 et «Notes \& Notebooks: Canada's Past in Pictures, (1-9) ", Bibliothèque et Archives Canada, Fonds Charles William Jefferys, MG30 D217 - Vol. 1.

${ }^{126}$ Robert Stacey, C.W. Jefferys 1869-1951, p. 7. Dans son ouvrage sur Jefferys, Stacey soutient que le peintre a renoncé à jouer un rôle actif "comme chef de file de l'école des paysagistes de Toronto dans les années 1920, laissant la place au Groupe des Sept et à ses disciples" (p. 9), il n'en demeura pas moins leur maître à penser, cherchant à faire découvrir la «nature véritable des paysages du Canada " (p. 7).

${ }^{127}$ Paul Walker, C.W. Jefferys and Images of Canadian Identity in School Textbooks, thèse de maîtrise, Queen's University, 1990, p. 2.

${ }^{128}$ Cité dans Ian E. Wilson, "Creating the future: Canada and Its Provinces", dans Yvan Lamonde, Patricia Fleming, Gilles Gallichan et Fiona A. Black, dir., 
Bien que Jefferys se soit permis quelques libertés, Papineau addressing meeting correspond largement au propos énoncé par Duclos DeCelles dans Papineau Cartier. Tant le visuel que l'écrit s'inscrivent dans le courant historique et idéologique de l'époque, qui reconnaissait les patriotes "comme artisans du gouvernement responsable et de l'indépendance du Canada ${ }^{129}$ ". En ajoutant la référence aux 92 résolutions et en campant l'assemblée devant une église, Jefferys rappelait non seulement l'origine historique de l'obtention de la responsabilité ministérielle et de la création d'une société démocratique, mais aussi que les Canadiens français avaient leur place au sein de la Confédération canadienne en tant que peuple fondateur. Enfin, en choisissant comme modèle l'église de Saint-Antoine, une paroisse qui ne connu aucune bataille, ni d'assemblée populaire, il ne faisait pas que reprendre un motif déjà connu et maîtrisé, il contribua à dédramatiser les insurrections.

Au terme de cette analyse d'œuvres réalisées entre 1837 et 1840 , on ne peut que souligner, encore une fois, que les perspectives qu'elles donnent à lire sur les rébellions relèvent autant de l'iconographie, de l'intention de l'artiste que de sa réception. Ainsi les bonnets phrygiens et la colonne apparentée à celle de Juillet peint par Charles Alexander ne sont probablement que de simples anachronismes, calques d'une production artistique parisienne et académique, de la part d'un artiste poursuivant sa formation au cœur de la France républicaine. Néanmoins, à une époque où l'influence de l'Église débordait largement sa fonction de guide spirituel, une telle présence ne pouvait qu'attiser la méfiance. L'œil attentif des détracteurs de l'héritage patriote reconnaissait dans cette apologie non seulement la volonté du peuple de se gouverner par lui-même, mais surtout

History of the Book in Canada: 1840-1918, Toronto, University of Toronto Press, 2005 , p. 175.

${ }^{129}$ Jean-Paul Bernard, Les Rébellions de 1837-1838, p. 32. 
l'affrontement entre le pouvoir civil et religieux qui, en France, avait finalement conduit le clergé à la marge du champ décisionnel régissant le sociopolitique.

Tout aussi audacieux que le Chénier ayant trouvé la mort lors de la bataille du 14 décembre 1837, celui de Louis-Philippe Hébert s'écartait des principes classiques qui seyaient généralement à la statutaire publique. Certes, les emprunts stylistiques à l'impressionnisme français permettaient de transposer dans la rigidité de la pierre toute la fougue et la détermination de celui qui avait combattu le pouvoir britannique, ce qu'une conception idéalisée du monument commémoratif excluait d'emblée. Or, cette représentation un peu trop réelle de ce patriote opposé au pouvoir impérial posait problème.' Dans un contexte où les relations extérieures du Canada étaient toujours dirigées par la mère patrie et où, au lendemain de l'affaire Riel et de la crise des écoles manitobaines, les Canadiens anglais remettaient en question la loyauté des Canadiens français à l'égard de la Grande-Bretagne, la représentation d'un Jean-Olivier Chénier plus grand que nature ne pouvait qu'alimenter la controverse sur la pertinence d'honorer la mémoire du héros de Saint-Eustache.

Quant à Papineau addressing meeting de Charles William Jefferys, contrairement aux œuvres précédentes, elle n’a provoqué aucune polémique, ni aucun passage au purgatoire, ni même de commentaire sur la décision de l'artiste de peindre une église dont le parvis n'avait pas été foulé par la contestation patriote. En fait, lorsque le public prit connaissance de l'œuvre, en 1912, le regard posé sur les rébellions avait beaucoup changé. D'une part, on reconnaissait aisément que la démocratie canadienne était redevable des revendications des patriotes bas-canadiens. D'autre part, les Canadiens français, qui pendant longtemps avaient remis en question la prise d'armes, convenaient que ce sacrifice avait permis de sauvegarder leur foi et leur langue ${ }^{130}$. Il est d'ailleurs intéressant de rappeler que le monument

${ }^{130}$ Déjà, en 1904, Robertine Barry en appelait dans sa nouvelle Le Patriote, parue dans L'Almanach du peuple Beauchemin, à la reconnaissance du sacrifice des 
élevé à la mémoire des patriotes de Saint-Denis l'année suivante attira lors de son inauguration le $1^{\text {er }}$ juillet, jour anniversaire de la Confédération, plus de 5000 personnes. Il n'est pas non plus anodin de penser que le slogan "Nos amis du Haut-Canada " ait été compris comme précurseur du bonententisme. Selon Robert Talbot, qui le situe entre 1916 et 1930, ce mouvement a été "lancé par un petit groupe d'anglophones ontariens $s^{131}$ " avec pour objectif d'encourager «le dialogue entre Canadiens français et Canadiens anglais ». L'œuvre elle-même, par le sujet traité et ses allusions à la naissance du pays, s'inscrivait dans la volonté de Jefferys de constituer un art authentiquement canadien.

Somme toute, ce regard posé sur quelques-unes des œuvres les plus importantes du corpus rébellien reste superficiel. Il y aurait encore beaucoup à questionner, à documenter et à mettre en relation avec le contexte de production ou celui de la réception de celles-ci. Ce n'est d'ailleurs que succinctement que les notions d'histoire, de mémoire et d'identité, pourtant convoquées dans ces représentations des rébellions, ont été abordées.

À titre d'exemple, issues toutes deux d'un regard d'aristocrate britannique, The Insurgent, At Beauharnois et les Lithographic Views ne posent absolument pas la question de l'identité sous le même angle. La première rend compte d'une possible perte identitaire, son statut d'aristocrate, alors que la seconde affirme une suprématie, celle des britanniques sur les colons canadiens. Même constat pour les dessins de Girouard et les aquarelles de Bouchette. Si les uns attestent l'affirmation de la présence canadienne en colonie anglaise, les autres

patriotes pour la survie du Canada français. On lira à ce sujet France St-Jean, Images du Patriote: objets commémoratifs, intentions variables, particulièrement le chapitre VIII, "Saint-Denis-sur-Richelieu, un lieu de mémoire des rébellions ou le lieu d'affirmation d'une identité nationale? ", de même que France St-Jean, "Saint-Denis-sur-Richelieu, lieu de mémoire des rébellions de 1837-1838: miroir d'une affirmation canadienne-française", dans Anne Gilbert, Michel Bock et Joseph-Yvon Thériault, dir., Entre lieux de mémoire : l'inscription de la francophonie canadienne dans la durée, Ottawa, Presses de l'Université d'Ottawa, 2009.

${ }^{131}$ Robert Talbot, "Une réconciliation insaisissable: le mouvement de la bonne entente, 1916-1930", Mens, vol. VIII, n 1 (automne 2007), p. 67-125. 
témoignent plutôt de la reconduction d'un modèle hiérarchique social. La lecture de Manifestation des Canadiens contre le gouvernement anglais, à Saint-Charles, en 1837, de la maquette du monument à Jean-Olivier Chénier et de Papineau addressing meeting montre bien que le résultat visuel de l'interprétation de l'artiste n'est pas que tributaire de son appartenance au Canada français ou au Canada anglais. D'autres référents identitaires sont convoqués ou conjugués, soit un à la France de 1789, dont le projet social ne fait pas consensus de ce côté-ci de l'Atlantique, un à l'Empire britannique, auquel, selon certains, loyauté est due, et un autre au Canada, pays en quête de sa propre identité.

De telles analyses n'empêcheront pas que l'on se réapproprie les œuvres afin qu'elles servent de nouveaux discours. Là n'est pas du tout mon objectif d'ailleurs. Néanmoins, cette analyse des œuvres, et plus particulièrement du vouloir dire qui y est inscrit, aura permis de démontrer que les représentations visuelles ne peuvent se résumer qu'à une simple évocation de la réalité historique ou à jouer le rôle de soutien illustratif. En tant que "miroir des préoccupations des sociétés qui les sécrètent ${ }^{132}$ ", elles sont tout autant document historique pouvant rendre compte des générations précédentes, que vecteur de l'imaginaire collectif.

${ }^{132}$ Pirotte, «Écoute Clio!... », p. 27. 